\title{
Chemical to Electrical Transduction using Fuel Cell Powered Organic Electrochemical Transistors
}

Siew Ting Melissa Tan ${ }^{1}$, Alexander Giovannitti ${ }^{1 *}$, Armantas Melianas ${ }^{1}$, Maximillian Moser ${ }^{2}$, Benjamin L. Cotts $^{1}$, Devan Singh ${ }^{1}$, Iain McCulloch ${ }^{2,3}$, Alberto Salleo ${ }^{1 *}$

${ }^{1}$ Department of Materials Science and Engineering, Stanford University

${ }^{2}$ Department of Chemistry, Imperial College London

${ }^{3}$ Physical Science and Engineering Division, King Abdullah University of Science and Technology

*Corresponding authors emails: ag19@stanford.edu and asalleo@stanford.edu

\begin{abstract}
The advancement of wearable biosensors is poised to change the landscape of personalized healthcare. Organic electrochemical transistors (OECTs) exhibit outstanding performance as metabolite sensors due to their biocompatibility and large transconductance. However, the conventional approach of conducting biochemical reactions within the electrolyte of the OECT can generate unintended side reactions and does not sufficiently take advantage of the amplification properties of the OECT. By isolating the reaction on a fuel cell, we harvest the energy from oxidoreductase enzymatic reactions of common metabolites to generate the gate potential biasing an OECT. This design fully leverages the OECT's large transconductance and renders the gate circuit self-powered using ambient energy sources. Utilizing this general approach, we can detect common metabolites comprising glucose, lactate or ethanol, and demonstrate large channel conductivity modulations with ON/OFF ratios up to $10^{4}$. This work introduces design rules for the development of high-gain self-powered biosensors.
\end{abstract}

\section{Introduction}

Organic mixed ionic-electronic conductors (OMIECs) are effective transducers of chemical to electronic information when used in electrochemical devices. OMIECs can accumulate electronic charges via chemical redox reactions while simultaneously being able to transport these charges along their conjugated backbone. Their operation in aqueous environments is further enabled by ionic transport via judicious side chain engineering ( $1-$ 3 ) or blending with polyelectrolytes (4). These unique properties enable their application in electrochemical devices, including bio-sensors $(5,6)$, actuators $(7)$, energy storage materials $(1)$, transistors $(8)$ and neuromorphic devices (9).

One device that leverages these properties of OMIECs is the organic electrochemical transistor (OECT) (10-12). The OECT is a three-terminal device that consists of a source, drain and gate electrode. The OMIEC is placed between the source and drain electrodes, defining the channel of the device, which is immersed in an electrolyte that provides ionic charge carriers. OECTs are often operated in aqueous electrolytes (12), ionic liquids or ion gels (13). Commonly, the gate electrode is an $\mathrm{Ag} / \mathrm{AgCl}$ pellet electrode that is immersed in an aqueous electrolyte. The OECT operates by modulating the electronic conductivity of the OMIEC with an external voltage source applying a potential, $V_{\mathrm{G}}$, between the gate and source electrodes to perform capacitive faradaic (14) charging (or discharging) reactions of the OMIEC. This process can be described as an electron-transfer (ET) reaction between the source and drain OECT electrodes and the OMIEC channel, during which ions simultaneously migrate into the OMIEC to electrostatically compensate for electronic charges on its backbone (15). The change in conductivity of the OMIEC is observed by measuring the drain current, $I_{\mathrm{D}}$, flowing in the channel upon application of a drain bias, $V_{\mathrm{D}}$, between the drain and source electrodes. Depending on the choice of OMIEC as the channel material, OECTs can exhibit large transconductances $\left(g_{m}=\frac{\partial I_{D}}{\partial V_{G}}\right)(12,16)$, allowing the OECT to amplify minute perturbations in gate potential into large modulations in channel conductivity.

The ability of OMIECs to interact with, transport, and store electronic and ionic charge carriers enable OECTs to record and transduce biological events in physiological conditions (5). Hence, OECTs have been widely investigated as biosensors (17-19) as well as for logic circuitry (20) where devices have been reported to be biocompatible $(21,22)$ and functional in aqueous environments (12). In addition to their outstanding transduction 
capabilities of in-vivo biological activity $(5,23)$, OECTs also demonstrate great potential for ex-vivo chemical sensing, such as the detection of metabolites facilitated by enzymatic reactions (24-28).

The detection of metabolites as biomarkers can provide early indication of disease onset, allowing for timely intervention before the appearance of aggravated symptoms. The specificity of enzymes affords targeted sensing of the biomarker of interest, leading to the widespread development of enzymatic biosensors. One example is the amperometric sensing of glucose using Glucose Oxidase enzymatic reactions for monitoring of Diabetes Mellitus $(28,29)$. Prior reports of OECT-based enzymatic sensors generally operate in two regimes as shown in Fig. 1 (A): (1) direct ET reactions from redox enzymes to the channel or gate electrode thus influencing the conductivity of the OMIEC $(26,30)$ or $(2)$ indirect ET via the formation of redox-active products such as hydrogen peroxide $\left(\mathrm{H}_{2} \mathrm{O}_{2}\right)$ that in turn modulate OECT performance (24). The reaction between the enzyme-metabolite couple and the OMIEC is a non-capacitive faradaic (14) electrochemical redox reaction between the reduced enzyme (direct ET) or $\mathrm{H}_{2} \mathrm{O}_{2}$ (indirect ET) and the OMIEC. These reactions lead to the reduction or oxidation of the OMIEC which modulates its conductivity (10), the metric utilized for sensing.

The majority of OECT enzymatic biosensors reported in literature perform these non-capacitive faradaic reactions within the electrolyte of the $\operatorname{OECT}(24,26,27,31)$, which poses significant challenges. Firstly, this approach fails to leverage the large transconductance that make OECTs suitable for amplifying sensed signals (32). Secondly, the operation mechanism of these devices can be complex due to simultaneous electrochemical side reactions (non-capacitive faradaic reactions) and the charging of the OMIEC channel material (capacitive faradaic reactions) during OECT operation. These confounding factors during multi-modal operation pose a significant challenge in understanding the physics of sensor performance, which impedes the rationalized development of these devices. Finally, achieving direct ET between the reduced enzyme and the OMIEC (Reaction 1, Fig. 1 (A)) requires careful alignment between the electronic energy levels of the OMIEC and the redox-active center of the enzyme (e.g. co-factor flavin adenine dinucleotide (FAD) in Glucose Oxidase) (33, 34). Other undesirable side reactions between the OMIEC and the electrolyte, including the oxygen reduction reaction (ORR) (Reaction 3) can occur, interfering with the sensing process and potentially degrading the OMIEC. This side reaction was reported to depend on the ionization potential (IP) of the OMIECs, the choice of the electrolyte, temperature and $\mathrm{pH}$ of the electrolyte (15). Poor ET between the enzymatic reaction and the OMIEC results in low reaction currents (nA), which require sophisticated measurement instrumentation to record.

Here, we propose a device design that spatially separates the enzymatic redox reactions on a fuel cell (noncapacitive faradaic reactions) from the operation of the OECT (capacitive faradaic reactions). The separation of the reaction from the transistor was previously implemented by the Frisbie group using ion gel-gated organic transistors (35). In our work, we employ Reaction 2 (Fig. 1 (A)) i.e. the formation of a redox intermediate $\mathrm{H}_{2} \mathrm{O}_{2}$ via oxidoreductase enzymatic reactions, to indirectly transfer charges from the metabolite and enzymatic reaction to the OMIEC. This approach avoids difficulties in achieving direct ET from the enzyme to the OMIEC due to the side reactions in ambient conditions that would interfere with sensing.

By utilizing the appropriate OMIEC as the OECT channel as well as the fuel cell electrode, we achieved large amplification of reaction currents of $10^{3}$ and up to four orders of magnitude modulation in $I_{\mathrm{D}}$ across the OECT channel. Furthermore, the use of OMIEC in the two components of the device (OECT and FC) of distinct operating modes (capacitive faradaic vs. non-capacitive faradaic) allows for improved understanding of the metabolite sensing mechanism based on enzymatic reactions using OECTs. By utilizing $\mathrm{H}_{2} \mathrm{O}_{2}$ as the oxidant for the fuel cell, the device can be generalized for many $\mathrm{H}_{2} \mathrm{O}_{2}$-producing enzymatic reactions such as glucose, lactate and ethanol, presenting a versatile approach for metabolite detection.

\section{$\underline{\text { Device concept }}$}

Figure 1 (B) illustrates the operation of the Fuel Cell OECT (FC-OECT). Unlike a conventional OECT where an external power source provides the $V_{\mathrm{G}}$ to perform charging/discharging reactions on the channel material, the $V_{\mathrm{G}}$ is generated by a chemical reaction within a two-electrode fuel cell (FC) consisting of an anode and cathode 
immersed in a shared electrolyte. The FC comprises an OMIEC that is a p-type conjugated polymer based on 3,3'-dialkoxybithiophene ( $\mathrm{p}(\mathrm{gT} 2))(1)$ as the anode. $\mathrm{p}(\mathrm{gT} 2)$ displays high electrochemical stability and rapid charging when used as the electrode material for water-based batteries (1), and was found to achieve reversible swelling during electrochemical charging and discharging in aqueous electrolytes (7). Electrodes can be prepared by processing the polymer from organic solvents to form water-insoluble thin films on conductive indium doped tin oxide (ITO) electrodes. As shown in Figure 1 (B), the polymer anode of the FC is in electrical contact with the $\mathrm{Ag} / \mathrm{AgCl}$ pellet immersed in a second electrolyte shared with the OECT channel. The counter electrode (cathode) of the FC is a bare ITO electrode that shares a common ground with the OECT source electrode. While the two electrochemical cells are physically separated, a Phosphate Buffered Saline (PBS) solution is used as the electrolyte of both cells. During operation of the device, metabolites (glucose, lactate or ethanol) and their corresponding oxidoreductase enzymes (Glucose Oxidase, Lactate Oxidase or Alcohol Oxidase) are added to the electrolyte in the FC compartment to produce $\mathrm{H}_{2} \mathrm{O}_{2}(36)$. The formation of $\mathrm{H}_{2} \mathrm{O}_{2}$ in turn oxidizes p(gT2) on the anode which changes its open circuit voltage (OCV) with respect to the grounded ITO cathode. The increase in OCV leads to concomitant redox reactions occurring on the $\mathrm{Ag} / \mathrm{AgCl}$ electrode leading to modulation of the conductivity of the OECT channel material. For an initially conductive p-type OECT channel material, the increase in gate potential can be utilized to discharge the channel material, thereby turning the OECT from the ON to the OFF state $(10)$.

\section{Results}

\section{$\underline{\text { Electrochemical characterization of the enzymatic reaction }}$}

The polymer p(gT2) is selected as the FC-anode since it oxidizes only partially in ambient conditions due to slow kinetics of the ORR in pH-neutral aqueous electrolytes. At the same time, p(gT2) is easily oxidized by $\mathrm{H}_{2} \mathrm{O}_{2}$ (hydrogen peroxide reduction reaction (HPRR), Reaction 2), which is intentionally formed during oxidoreductase reactions of metabolites as it is the reactant of interest. $\mathrm{p}(\mathrm{gT} 2)$ polymer electrodes were fabricated by spin coating on ITO-coated glass substrates. The chemical oxidation (positive charging) of $\mathrm{p}(\mathrm{gT} 2)$ by $\mathrm{H}_{2} \mathrm{O}_{2}$ in ambient conditions was monitored using three-electrode electrochemical measurements by measuring the potential generated between the $\mathrm{p}(\mathrm{gT} 2)$-coated ITO electrode and a saturated $\mathrm{Ag} / \mathrm{AgCl}$ reference electrode.

Fig. 2 (A) presents the change in the OCV of the anode after addition of glucose to Glucose Oxidase (GOx) in PBS electrolyte (red trace). As expected, an increase of the OCV is observed due to the HPRR showing an increase of more than $200 \mathrm{mV}$ over a time span of 20 minutes. The formation of $\mathrm{H}_{2} \mathrm{O}_{2}$ was verified by Peroxidase enzymatic reactions and a dye (37) (Fig. S1). A control experiment was conducted by adding an equivalent concentration of $\mathrm{H}_{2} \mathrm{O}_{2}$ to PBS, resulting in rapid oxidation of the polymer (Fig. 2A, blue trace). The rapid charging shows that HPRR occurs spontaneously and that the rate of the charging of p(gT2) is limited by the rate of the enzymatic reaction, which is dependent on the enzymatic activity of GOx. We also investigated mass transport limitations arising from different concentrations of glucose in the solution (Fig. S2). While an increase in the glucose concentration is expected to enhance the rate of $\mathrm{p}(\mathrm{gT} 2)$ oxidation, a concomitant increase in solution viscosity limits mass transport. We thus used a $33 \mathrm{mM}$ glucose solution (in PBS) throughout this work as we found it to be the optimum glucose concentration for rapid $\mathrm{H}_{2} \mathrm{O}_{2}$ production.

In addition to a substantial increment of the OCV, the magnitude of the reaction current generated on the fuel cell should be larger or equal to the typical gate currents required to gate an OECT. This is due to the fact that enough charges must be injected into the OECT channel to sustain its change in conductivity. To quantify the catalytic activity of $\mathrm{p}(\mathrm{gT} 2)$ towards HPRR, chronoamperometry (CA) measurements were conducted to determine the cumulative charge (holes) injected into the polymer during the reaction (Fig. 2B). The electrons injected into the p(gT2) electrode by the potentiostat compensate for the holes injected into the polymer due to HPRR to maintain a constant OCV. The accumulated charge injected (normalized by area) is shown in Fig. 2B and Fig. S3 with currents of 1-2 $\mu \mathrm{A}$. The increment in electrons injected into $\mathrm{p}(\mathrm{gT} 2)$ after performing the enzymatic reaction with glucose (red) or the addition of $\mathrm{H}_{2} \mathrm{O}_{2}$ (blue) further demonstrates that $\mathrm{p}(\mathrm{gT} 2$ ) facilitates HPRR. To further understand $\mathrm{p}(\mathrm{gT} 2)$ 's catalytic activity for HPRR, cyclic voltammetry (CV) measurements were conducted (see 
Fig. S4). The electrochemical measurements demonstrate that indirect ET occurs between glucose/GOx and $\mathrm{p}(\mathrm{gT} 2)$ (via the intermediate $\mathrm{H}_{2} \mathrm{O}_{2}$ ) while no direct ET from GOx to $\mathrm{p}(\mathrm{gT} 2)$ is observed.

Another method to verify the oxidation of $\mathrm{p}(\mathrm{gT} 2)$ is by characterization of the optical changes of $\mathrm{p}(\mathrm{gT} 2)$ after exposing it to electrolytes that contain glucose and GOx. OMIECs based on conjugated polymers appear colored due to optical transitions between the HOMO and LUMO. Oxidation of a p-type polymer such as p(gT2) leads to a decrease in the neutral chain optical absorption and the formation of polarons with distinct absorption spectra at lower energy, as reported in the literature (38). Fig. 2 (C) shows the thin film absorption spectrum of the pristine $\mathrm{p}(\mathrm{gT} 2)$ that has a broad absorption peak between $450 \mathrm{~nm}$ and $800 \mathrm{~nm}$. After treatment of a $\mathrm{p}(\mathrm{gT} 2)$ thin film with the glucose/GOx electrolyte and upon oxidation of the polymer, the intensity of the absorption peak $(\lambda \max =650$ $\mathrm{nm}$ ) decreases while a new absorption peak appears at longer wavelengths, indicating polaron formation (1). We conducted spectroelectrochemical measurements to verify the changes of the absorption spectrum when the $\mathrm{p}(\mathrm{gT} 2)$ is electrochemically charged/discharged in PBS electrolyte (Fig. S5).

\section{$\underline{\text { Performance of Fuel Cell-OECT }}$}

The increase in potential across the fuel cell due to the oxidation of $\mathrm{p}(\mathrm{gT} 2)$ is used to gate a p-type depletion mode OECT to turn it from ON to OFF. To maximize the performance of the FC-OECT (i.e. the magnitude of $I_{\mathrm{D}}$ modulation), the range of potentials generated across the $\mathrm{FC}$ should have large overlap with the gate potentials $\left(V_{\mathrm{G}}\right)$ at which the OECT exhibits the greatest transconductance $\left(g_{\mathrm{m}}\right)$. Based on this criterion, $\mathrm{p}(\mathrm{gT} 2)$ was also selected as the material for the OECT channel. When exposed to aqueous electrolytes in ambient conditions, p(gT2) undergoes ORR and becomes partially oxidized, increasing its conductivity, as previously reported for electron-rich polythiophenes (15). Thus, p(gT2) can be operated as a depletion mode OECT in ambient conditions. Characteristics of the OECT using p(gT2) are shown in Fig. S6.

Fig. 3 (A) shows the performance of the $\mathrm{p}(\mathrm{gT} 2) \mathrm{FC}-\mathrm{p}(\mathrm{gT} 2)$ OECT when the glucose/GOx reaction is performed on the FC. Upon addition of glucose to a solution of GOx, the potential ( $E_{\mathrm{WE}}, \mathrm{p}(\mathrm{gT} 2)$ working electrode vs ITO counter electrode) across the FC increases due to oxidation of $\mathrm{p}(\mathrm{gT} 2)$ by $\mathrm{H}_{2} \mathrm{O}_{2}$ generated by the enzymatic reaction. This increase in gate bias results in discharging of $\mathrm{p}(\mathrm{gT} 2)$ in the OECT channel and thus a decrease in source-drain current magnitude $\left(I_{\mathrm{D}}\right)$. In a control experiment, an equivalent concentration of $\mathrm{H}_{2} \mathrm{O}_{2}$ was added to the PBS electrolyte of the FC, leading to a rapid rise in $E_{\mathrm{WE}}$ and decrease in $I_{\mathrm{D}}$ (Fig. $3(\mathrm{~B})$ ), as expected. Increasing the concentration of the analyte (glucose or $\mathrm{H}_{2} \mathrm{O}_{2}$ ) in the electrolyte of the fuel cell increases the rate of charging of the polymer, and thus the rate of rise in $E_{\mathrm{WE}}$ and drop in $I_{\mathrm{D}}$ (Fig. S7), showing the direct relationship between the concentration of $\mathrm{H}_{2} \mathrm{O}_{2}$ and rate of charging of the polymer on the FC. To verify the efficacy of gating the OECT by the enzymatic reaction at the FC, the transfer curve of the OECT (inset) was fitted to a polynomial function and the function applied to the time-varying evolution of $E_{\mathrm{WE}}$ to calculate the expected $I_{\mathrm{D}}$ (red dotted lines). The experimental and calculated $I_{\mathrm{D}}$ agree well across the majority of our devices, indicating that the power output from the chemical reaction on the FC is indeed sufficient to gate the OECT.

We further demonstrate that this device can be generalized to other $\mathrm{H}_{2} \mathrm{O}_{2}$-producing oxidoreductase enzymatic reactions such as the oxidation of lactate by Lactate Oxidase (LOx) and ethanol by Alcohol Oxidase (AlOx) (Fig. S8) which are important biomarkers for health monitoring. The reaction of lactate induced more than two orders of magnitude modulation in $I_{\mathrm{D}}$. Depending on the type of enzyme and the concentration of active units in solution, the rate of $\mathrm{H}_{2} \mathrm{O}_{2}$ formation, $E_{\mathrm{WE}}$ increase of the $\mathrm{FC}$, and decrease of $I_{\mathrm{D}}$ can vary.

\section{Mechanism of FC-OECT operation}

Further development of FC-OECT devices requires elucidation of the device operation mechanism as well as materials design principles for the two electrochemical cells. We postulate that the working principle of the FCOECT is dependent on the relationship between redox reactions on the $\mathrm{p}(\mathrm{gT} 2)$-coated ITO electrode and on the $\mathrm{Ag} / \mathrm{AgCl}$ pellet. 
The $\mathrm{Ag} / \mathrm{AgCl}$ pellet that is commonly used as the gate electrode in the OECT serves as a non-polarizable pseudoreference electrode. Applying gate potentials within a certain window $(-0.1 \mathrm{~V}$ to $+0.1 \mathrm{~V}$ in $\mathrm{pH}$ neutral electrolyte) leads to redox reactions between the electrolyte and $\mathrm{Ag} / \mathrm{AgCl}$. This is evident in Fig. S9 which shows peaks in gate current between $-0.1 \mathrm{~V}$ to $+0.1 \mathrm{~V}$ during measurements of the OECT transfer curve, which are not observed for a polarizable gate electrode such as $\mathrm{Pt}$. The non-polarizable nature of the $\mathrm{Ag} / \mathrm{AgCl}$ gate minimizes the potential drop across the $\mathrm{Ag} / \mathrm{AgCl}$ pellet and allows for the majority of the potential drop to occur across the OMIEC channel, resulting in effective charging/discharging of the channel. We thus hypothesize that an increase in potential across the $\mathrm{FC}$ within the range of $-0.1 \mathrm{~V}$ to $+0.1 \mathrm{~V}$ vs $\mathrm{Ag} / \mathrm{AgCl}$ leads to the oxidation of $\mathrm{Ag}$ to $\mathrm{Ag}^{+}$ where $\mathrm{AgCl}$ is formed. Simultaneously, an electron is injected into the OECT channel while chloride ions are released from the channel to maintain electroneutrality of the electrolyte. Hence, $\mathrm{p}(\mathrm{gT} 2)$ in the OECT channel becomes reduced and a decrease in channel conductivity is observed.

Due to the competing side-reactions (ORR) occurring on the discharged, neutral $\mathrm{p}(\mathrm{gT} 2)$, a minimum of $\sim 5 \mathrm{nA}$ of continuous gate current is required to sustain the discharge of the OECT channel as shown in Fig. S9. Hence, the reaction currents generated at the FC must be large enough to overcome the side reactions that occur in ambient conditions. The reaction currents achieved on the FC are 1-2 $\mu \mathrm{A}$ (Fig. S3), and thus sufficient to sustain the gating of the OECT. Due to the ORR on the OECT channel, $I_{D}$ reverts to its initial value within several seconds upon disconnecting the two electrochemical cells (Fig. 3 (B)). While ORR reactions on the OECT channel are generally undesirable due to increased gate current required and $\mathrm{H}_{2} \mathrm{O}_{2}$ formation during device operation (15), we instead utilize the ORR to our benefit in the operation of the FC-OECT. ORR allows the OECT to recover its initial state (ON) after the FC is disconnected from OECT gate, allowing the OECT to be reused. In the absence of ORR, a negative $V_{\mathrm{G}}$ would have to be applied across the OECT to re-oxidize the channel to recover its initial conductive state.

OMIECs are the prime material choice for the FC due to their catalytic activity for HPRR, as well as their ability to perform volumetric charging reactions in the presence of $\mathrm{H}_{2} \mathrm{O}_{2}$-containing electrolytes. As shown in Fig. S10, the reaction current, or amount of charge injected into the $\mathrm{p}(\mathrm{gT} 2) \mathrm{FC}$ increases with film thickness. Hence, we postulate that the charging of the electrode begins at the polymer/electrolyte interface due to their proximity to the reactants. The introduced holes are compensated by anions provided by the electrolyte, that often carry hydration shells, which increase the swelling of the polymer during charging. In addition, $\mathrm{H}_{2} \mathrm{O}_{2}$ 's chemical similarity with $\mathrm{H}_{2} \mathrm{O}$ (ability to form hydrogen bonds with the ethylene glycol side chains) could allow it to be transported into the bulk of the polymer in a similar manner as the latter and thus facilitate the volumetric charging of the electrode. Unlike planar electrodes where reactions occur on the surface of the electrode, the volumetric charging and mobility of charges within OMIECs allow for reactants to rapidly permeate and react throughout the bulk of the material, enabling the high gate currents needed to operate the OECT.

\section{$\underline{\text { Optimization of FC and OECT channel materials }}$}

To further understand the operation mechanism of the FC-OECT and improve its performance, we investigated several materials for the FC and OECT channel. We first discuss the choice of OMIECs for the FC anode by studying the influence of their electronic energy levels on performing HPRR. Next, we investigate the functionalization of the FC counter electrode (cathode) to enhance the potential increment of the FC electrode. Finally, we elucidate the choice of the OECT channel material to maximize overall device performance.

\section{$\underline{\text { 1. Material selection for FC anode }}$}

The effectiveness of the fuel cell anode material in transducing the presence of $\mathrm{H}_{2} \mathrm{O}_{2}$ into an electrical signal depends on its thermodynamic driving force and kinetic ability to drive the HPRR. This in turn influences the rate and extent of oxidation of the material to generate a potential at the gate electrode for OECT operation. It is important to consider the electronic energy levels of the anode material relative to the electrochemical potential of electrons in the reactants $\left(\mathrm{H}_{2} \mathrm{O}_{2}\right.$ (HPRR) or $\mathrm{O}_{2}(\mathrm{ORR})$ ). The material should be resistant to ORR to avoid undesired spontaneous charging so that it is stable at ambient conditions, while readily promoting HPRR, the 
reaction of interest. For effective operation of the FC-OECT, we hypothesize that the potential generated on the anode should be sufficiently large to drive the oxidation of the $\mathrm{Ag} / \mathrm{AgCl}$ pellet electrode of the OECT $(-0.1 \mathrm{~V}$ to $+0.1 \mathrm{~V})$, and to provide enough current to maintain the discharged state of the OECT material.

We compare the properties of $\mathrm{p}(\mathrm{gT} 2)$ to other state-of-the-art polymers for OECTs. This includes PEDOT:PSS, a conducting polymer, usually employed in depletion mode OECTs and p(gPyDPP-MeOT2), a semiconducting polymer used in enhancement mode OECTs (15). Fig. 4 (A) illustrates the energy levels of the polymers (Ionization Potentials) in relation to the electrochemical potentials at which HPRR $\left(\mathrm{H}_{2} \mathrm{O}_{2}\right)$ or ORR $\left(\mathrm{O}_{2}\right)$ occur under in $\mathrm{pH}$ neutral aqueous electrolytes. PEDOT:PSS has the highest HOMO (4.0 eV), followed by $\mathrm{p}(\mathrm{gT} 2)$ $(4.5 \mathrm{eV}(1))$ and $\mathrm{p}(\mathrm{gPyDPP}-\mathrm{MeOT} 2)(5.0 \mathrm{eV}(15))$. This comparison aids in the elucidation of the optimal energy levels of the anode material for the operation of the FC in ambient conditions.

Fig. 4 (B) shows the change in $\mathrm{OCV}$ vs. saturated $\mathrm{Ag} / \mathrm{AgCl}$ of the three polymers on ITO coated glass substrates after addition of glucose to a GOx solution. A significant increase in the OCV of p(gT2) and p(gPyDPP-MeOT2) is observed, indicating oxidation of the polymers via HPRR, while the OCV of PEDOT:PSS is barely perturbed by the reaction.

This observation can be explained by comparing the polymers' IP as well as their degree of charging in ambient conditions. Since PEDOT:PSS has the smallest IP of the polymer series in its neutral, discharged form, it reacts with molecular oxygen during ORR to become highly oxidized in ambient conditions (15). Hence, there is little driving force for PEDOT:PSS to undergo further HPRR to reach an additional degree of charging in ambient conditions, rendering it unsuitable to function as the anode. In contrast, the larger IPs of p(gT2) and p(gPyDPPMeOT2) result in lower propensity towards ORR, allowing $\mathrm{p}(\mathrm{gT} 2)$ to only partially oxidize and $\mathrm{p}(\mathrm{gPyDPP}-$ MeOT2) to be mostly neutral in ambient conditions (15) as evident from their lower initial OCV compared to PEDOT:PSS. Since the potential for the HPRR $(+1.1 \mathrm{~V}$ vs $\mathrm{Ag} / \mathrm{AgCl})$ is significantly deeper than the IP of these materials $\left(-0.2 \mathrm{~V}\right.$ and $+0.3 \mathrm{~V}$ vs $\mathrm{Ag} / \mathrm{AgCl}$ respectively), both polymers can be oxidized by $\mathrm{H}_{2} \mathrm{O}_{2}$. Due to the greater difference between the oxidation potential for $\mathrm{p}(\mathrm{gT} 2)$ to potentials at which HPRR occur, the thermodynamic driving force for HPRR by $\mathrm{p}(\mathrm{gT} 2)$ is expected to be greater than that of $\mathrm{p}(\mathrm{gPyDPP}-\mathrm{MeOT} 2)$. The catalytic activities of $\mathrm{p}(\mathrm{gT} 2)$ and $\mathrm{p}(\mathrm{gPyDPP}-\mathrm{MeOT} 2)$ towards HPRR were further investigated using CA (Fig. 4 (C)). For thin films of similar film volume, $\mathrm{p}(\mathrm{gT} 2)$ exhibits two orders of magnitude larger current density than p(gPyDPP-MeOT2) during the HPRR, whereas PEDOT:PSS, as expected, showed minimal reaction current (Fig. $\mathrm{S} 11)$.

While p(gPyDPP-MeOT2) exhibits a rapid increase in OCV, in fact only a small amount of charges is injected into the polymer. This result can be understood by overlaying the change in OCV onto the $\mathrm{CV}$ of $\mathrm{p}(\mathrm{gPyDPP}-$ MeOT2) (Fig. 4 (D)). During HPRR, very few electronic states are involved in the reaction, which can be attributed to the oxidation of the most well-aligned polymer chains only, due to their lower oxidation potential. On the other hand, more charges are injected into p(gT2) as illustrated by the grey shaded area in Fig. 4 (D). Maximizing the amount of charges generated on the FC electrode is needed in order to sustain the gating of the OECT, as discussed in the above sections.

Our results indicate that a useful material for the anode must not only have appropriate energy levels but also sufficient reactive states that can perform HPRR. Based on this analysis, we thus focused our work on p(gT2) as the material of choice for the anode. When compared to $\mathrm{p}(\mathrm{gPyDPP}-\mathrm{MeOT} 2), \mathrm{p}(\mathrm{gT} 2)$ achieved higher reaction current densities, leading to greater $I_{\mathrm{D}}$ modulations of the FC-OECT as shown in Table S1. 
In our device architecture thus far, a bare ITO electrode serves as a passive counter electrode (cathode) that references the FC to a common ground with the OECT. We postulate that there is negligible direct or indirect electron transfer between $\mathrm{GOx}$ or $\mathrm{H}_{2} \mathrm{O}_{2}$ to the bare ITO so the potential increase across the reaction cell is solely due to the oxidation of $\mathrm{p}(\mathrm{gT} 2)$. The overall change in potential can be further improved by functionalizing the counter electrode (Fig. 5 (A)) with a material that has an appropriate energy level to receive electrons directly from the enzyme.

To enable spontaneous direct ET from the GOx to the cathode, their energy levels need to be aligned, requiring cathode materials of large electron affinities (EAs). The complex tetrathiafulvalene tetracyanoquinodimethane (TTF-TCNQ, Fig. S12) was selected as an appropriate material since it was reported as an effective electron mediator with GOx due to the high EA of TCNQ ${ }^{-}$of $4.55 \mathrm{eV}(34)$ with respect to the reduced $\mathrm{FADH}_{2} \mathrm{Co}^{-\mathrm{factor}}$ of GOx (4.38 eV) (34). TTF-TCNQ is a conductive complex that can be solution processed by dispersing it in acetonitrile and drop casting onto ITO electrodes. As illustrated in Fig. 5 (B), the electron extracted from the oxidation of glucose to reduce FAD to $\mathrm{FADH}_{2}$ can undergo two separate pathways: (Pathway 1) direct ET to the electron mediator TTF-TCNQ or (Pathway 2) reduction of $\mathrm{O}_{2}$ to form $\mathrm{H}_{2} \mathrm{O}_{2}$ which oxidizes $\mathrm{p}(\mathrm{gT2}$ ). The bifunctionalized fuel cell using TTF-TCNQ and p(gT2) exhibited a potential change $>300 \mathrm{mV}$ (Fig. 5 (C)) that indicates direct ET from the reduced enzyme to the cathode. The total potential change across the TTF-TCNQ $\mathrm{p}(\mathrm{gT} 2)$ cell is approximately the sum of the potential changes of $\mathrm{p}(\mathrm{gT2})$ vs ITO (purple) and TTF-TCNQ vs ITO (green). Utilization of the bi-functionalized FC in the FC-OECT led to an increase in the ON/OFF ratio of the OECT of up to four orders of magnitude, distinctly turning the device from an ON to an OFF state with low OFF currents of $30 \mathrm{nA}$ (Fig. 5 (D)). To our knowledge, this large change in channel conductance has yet to be achieved by OECT-based biosensors. An alternative approach to achieving direct electron transfer with OMIECs was recently reported (28) for OMIECs based on n-type NDI copolymers (39). When tested in our FC-OECT setup, we instead observed that the electron was rapidly transferred to $\mathrm{O}_{2}$, resulting in $\mathrm{H}_{2} \mathrm{O}_{2}$ formation instead of electron transfer to the NDI-copolymer (Fig. S13).

\section{Materials and device dimensions of OECT}

To maximize the magnitude of $I_{\mathrm{D}}$ modulation of the OECT, the range of potential increase across the fuel cell should ideally overlap with the OECT gate potential $\left(V_{\mathrm{G}}\right)$ at which $g_{\mathrm{m}}$ is maximized, which can be deduced from its transfer characteristics. Furthermore, to achieve the largest ON/OFF ratios, the potential change on the FC should enclose the OECT's subthreshold region as well (i.e. centered around the turn on voltage $\left(V_{\mathrm{T}}\right)$ ). To achieve this goal, we varied the channel material (16) and aspect ratio (40) $(\mathrm{W} / \mathrm{L})$ of the OECT which have been previously shown to be effective for modifying the characteristics of OECTs. In addition to p(gT2), we investigated stateof-the-art OECT materials p(g2T-TT) (2) and PEDOT:PSS as candidates for depletion mode OECT channel materials. These polymers were selected based on their differences in their operational characteristics $\left(V_{\mathrm{T}}\right.$ and $\left.g_{\mathrm{m}}\right)$. PEDOT:PSS with the shallowest IP, has the largest $V_{\mathrm{T}}(+0.6 \mathrm{~V}$ vs $\mathrm{Ag} / \mathrm{AgCl})$, followed by $\mathrm{p}(\mathrm{g} 2 \mathrm{~T}-\mathrm{TT})(+0.2$ $\mathrm{V}$ ), and p(gT2) (0 V) (Fig. S14). Comparing their transfer characteristics (potentials at which $g_{\mathrm{m}}$ is maximized) with the range of potential changes on the FC, we determined that the OECT using p(gT2) with an aspect ratio of $W / L=200$ was the most optimal (Fig. S6). This design was further verified by FC-OECT measurements summarized in Table S2 where OECTs with $\mathrm{p}(\mathrm{gT} 2)$ channel achieved the largest $I_{\mathrm{D}}$ modulations followed by $\mathrm{p}(\mathrm{g} 2 \mathrm{~T}-\mathrm{TT})$ and PEDOT:PSS.

Optimizing the fuel cell and OECT individually streamlines the device development process. Not only does p(gT2) fulfil the individual design criteria of both the fuel cell's anode and OECT channel, utilizing the same polymer for both components ensures their similarity in redox and electronic characters. Utilizing identical materials for both the FC anode and OECT channel also simplifies the device fabrication process.

\section{Towards the ideal FC-OECT}


While the performance of the current FC-OECT is encouraging for applications in biosensors, we acknowledge limitations and challenges that should be addressed in future device design. Firstly, limiting ORR on the FC anode as much as possible in ambient conditions will minimize drift of the initial potential of the FC, while maximizing the density of electronic states accessible for oxidation by HPRR. This could be achieved by designing an airstable p-type polymer with $4.5 \mathrm{eV}<\mathrm{IP}<5 \mathrm{eV}$. Secondly, the counter electrode should comprise ideally of a solution-processable n-type polymer that has an EA larger than that of $\mathrm{FADH}_{2}$ for efficient direct ET from the enzyme to the cathode. One way to lower the EA of $n$-type polymers is to substitute protons of the polymer backbone with electron-withdrawing atoms or groups (41) or select polymer backbones that have large EAs (42, 43).

Currently, the rate of potential change on the FC is limited by the kinetics of the enzymatic reaction. To improve reaction kinetics at both electrodes, enzymes can be immobilized onto the electrodes either by entrapment between polymer chains (44) or by direct covalent bonding between amino acid residues of the enzyme with the side chains of the polymer (45). This bi-functionalized fuel cell performs analogously to a biofuel cell where chemicallydriven opposing redox reactions are conducted on two electrodes to generate a potential difference and reaction current. Hence, further optimization of the fuel cell could draw inspiration from the well-established field of biofuel cells.

Lastly, the OECT can be further improved by improving the mobility of the channel material to achieve higher $g_{\mathrm{m}}$ and by designing materials with larger OECT ON/OFF ratios.

\section{Discussion}

We have presented a versatile method for biosensing that challenges the conventional approach of conducting sensing reactions within the electrolyte of an OECT. The large amplification properties of OECTs $\left(g_{\mathrm{m}}\right)$ leverages externally applied potentiometric modulations between the gate and channel to induce changes in channel conductivity, rather than amperometric changes from direct interaction between analytes and the OECT channel. When operated amperometrically, electron transfer can occur between the analyte with both the gate and channel which is akin to a gate current leakage. The application of gate and drain bias further complicates the operation of the sensor as the redox reaction kinetics are electric field dependent. Hence, it is in general not straightforward to operate an OECT biosensor amperometrically. The fuel cell operating alone as an amperometric sensor is limited by the charge output by the enzymatic reaction and is only able to generate up to $\sim 1-2 \mu \mathrm{A}$. However, if the fuel cell is operated potentiometrically by utilizing its change in potential to gate the OECT, the gate current is amplified by $10^{3}$ to $\sim 1-2 \mathrm{~mA}$ in $I_{\mathrm{D}}$ with up to $10^{4}$ change in $I_{\mathrm{D}}$. This impressive degree of current amplification and magnitude of $I_{\mathrm{D}}$ modulation has yet to be achieved by conventional OECT biosensors which conduct the reaction within the electrolyte of the OECT. Achieving currents on the order of $\mathrm{mA}$ further simplifies the measurement instrumentation required for signal recording, in contrast to OECT biosensors that operate in the nA regime.

Furthermore, by isolating the reaction on the fuel cell, the FC-OECT can be made modular, where the OECT can be reused for different reactions by swapping in a reaction cell with the desired enzyme. In addition to other $\mathrm{H}_{2} \mathrm{O}_{2}$-producing reactions, the device could be extended to gas sensors that detect other strong oxidizers such as other peroxides or reactive gases such as halogens or nitrogen oxides, depending on the relative energy levels of these redox-active chemicals and the electronic energy levels of the anode polymer. More generally, if the twoelectrode cell can transduce an external stimulus into sub-volt potential changes, it can be utilized to gate an OECT. This opens possibilities of implementing a myriad of devices as the transduction cell ranging from optoelectronic devices to piezoelectric pressure sensors (46).

By harvesting the chemical energy from spontaneous enzymatic reactions of metabolites to generate a gate potential, only a single low power source is required to probe the change in conductivity of the channel. Hence, in future developments, the device can be integrated with a battery onto a single platform, potentially allowing it to be fully autonomous. This greatly simplifies the design and operation of next-generation OECT-based devices. 
The combined ability to convert the chemical energy of metabolites into an electrical signal as well as perform simple analog-to-digital computation opens further possibilities of the FC-OECT's application in low-powered biocomputing with localized sense-compute-treat capabilities. 


\section{Materials and Methods}

$\underline{\text { Materials }}$

Polymers p(gT2) (1), p(g2T-TT) (2) and p(gPyDPP-MeOT2) (15) were synthesized according to the literature. 3,3,5,5-Tetramethylbenzidine, Horseradish Peroxidase Type VI from Amoracia rusticana, Glucose Oxidase (GOx) type II from Aspergillus niger, Lactate Oxidase (LOx) from Aerococcus viridans, and Alcohol Oxidase (AlOx) from Hansenula sp. were purchased from Sigma-Aldrich and stored at $-20^{\circ} \mathrm{C}$. Clevios $\mathrm{PH} 1000$ PEDOT:PSS solution was purchased from Heraeus and stored at $4{ }^{\circ} \mathrm{C}$. $30 \% \mathrm{H}_{2} \mathrm{O}_{2}$ solution in $\mathrm{H}_{2} \mathrm{O}$ was purchased from Sigma-Aldrich and stored at $4^{\circ} \mathrm{C}$. D-Glucose, Sodium Lactate, and TTF-TCNQ were purchased from Sigma-Aldrich. PDMS adhesives were purchased from 3M. $2 \mathrm{~cm} \times 2 \mathrm{~cm}$ polished indium tin oxide (ITO) coated glass slides for the fuel cell were purchased from Xin Yang Technology Ltd.

Fuel Cell anode preparation

$\mathrm{p}(\mathrm{gT} 2)$ and $\mathrm{p}(\mathrm{gPyDPP}-\mathrm{MeOT} 2)$ were dissolved in chloroform at $10 \mathrm{mg} / \mathrm{mL}$ and spin coated on ITO/glass at 1000 $\mathrm{rpm}$, with the exception of $\mathrm{p}(\mathrm{gT} 2)$ thickness variation samples in the SI, and annealed at $60^{\circ} \mathrm{C}$ for 10 minutes to yield film thickness of $120 \mathrm{~nm}$ and $150 \mathrm{~nm}$, respectively. PEDOT:PSS films were prepared by spin coating on ITO/glass a solution of Clevios PH 1000 with 6\% ethylene glycol, 1\% (3-glycidyloxypropyl)trimethoxysilane, dodecylbenzene sulfonic acid and annealed at $120^{\circ} \mathrm{C}$ for 20 minutes to yield $200 \mathrm{~nm}$ films (47).

\section{OECT fabrication}

Photolithographically patterned chips with gold contacts and patterned Parylene to expose regions for the channel were used as OECT chips (12). Channel dimensions were $W=2 \mathrm{~mm}, L=10,20,100$ and $200 \mu \mathrm{m}$. $\mathrm{p}(\mathrm{gT} 2)$ and $\mathrm{p}(\mathrm{g} 2 \mathrm{~T}-\mathrm{TT})$ were dissolved in chloroform at $10 \mathrm{mg} / \mathrm{mL}$ and spin coated at $1000 \mathrm{rpm}$, annealed at $60^{\circ} \mathrm{C}$ for 10 minutes, followed by parylene lift-off to form the channel. PEDOT:PSS OECTs were prepared in similar method and from similar recipe as the fuel cell anode.

\section{Film thickness measurements}

Thickness measurements were conducted on a DekTak 150 Surface Profiler using $1 \mathrm{mg}$ tip force.

\section{Enzyme solutions}

Enzyme solutions were prepared in the concentrations of $20.4 \mathrm{mg} / \mathrm{mL}, 2.4 \mathrm{mg} / \mathrm{mL}$ and $14 \mathrm{mg} / \mathrm{mL}$ in phosphate buffered saline solution for GOx, LOx and AlOx respectively. Fresh solutions were prepared before each measurement.

$\underline{\text { Viscosity measurements }}$

Viscosity measurements of varying concentrations of glucose (10, 30, 50, 100 and $1000 \mathrm{mM})$ dissolved in PBS were conducted on a TA Instrument ARES-G2 using a cup and bob setup at $25^{\circ} \mathrm{C}$.

\section{Electrochemical measurements}

All electrochemical characterization of the fuel cell anode or cathode were conducted using a 3-electrode setup with a saturated $\mathrm{Ag} / \mathrm{AgCl}$ reference (BASi) and Pt mesh counter electrode on a Biologic SP-300. PDMS wells were adhered on the fuel cell substrates to confine the reaction solution. For monitoring the open circuit potential between the polymer and $\mathrm{Ag} / \mathrm{AgCl}$ reference using enzymatic reactions, $400 \mu \mathrm{L}$ of enzyme solution was drop cast on the surface of the fuel cell and $200 \mu \mathrm{L}$ of $100 \mathrm{mM}$ analyte added after 1 minute. For control measurements, $400 \mu \mathrm{L}$ of PBS was drop cast and $200 \mu \mathrm{L}$ of $100 \mathrm{mM} \mathrm{H}_{2} \mathrm{O}_{2}$ added after 1 minute. In chronoamperometry measurements, the potential of the working electrode was held at its initial open circuit potential and the current flow into the film measured during the above reactions. The scan rate for the cyclic-voltammogram measurements was $50 \mathrm{mV} / \mathrm{s}$ and conducted in phosphate buffered saline solution unless stated otherwise.

\section{Spectroelectrochemical measurements}

A custom-built optical and electrochemical setup using Ocean Optics 35 Red Tide Spectrometer, halogen lamp, and an Ivium potentiostat was used to obtain the spectral changes of polymer films in PBS as potential was applied to the film. Cyclic--voltammetry was conducted using a three-electrode setup in a custom-built quartz cuvette cell (Starna cells) at $50 \mathrm{mV} / \mathrm{s}$ and an average of 5 spectra taken every $1 \mathrm{~s}$.

\section{$\underline{\mathrm{UV}-\mathrm{vis} \text { measurements of thin films }}$}

UV-vis spectrum of a pristine $165 \mathrm{~nm}$ thick film of $\mathrm{p}(\mathrm{gT} 2)$ on ITO coated glass was taken using a Cary 6000i UV-Vis-NIR. The film was immersed in a solution of $10 \mathrm{~mL}$ GOx and $5 \mathrm{~mL} 100 \mathrm{mM}$ Glucose solution in PBS for 20 minutes before rinsing with DI water and taking another spectrum. 
$1 \mathrm{mg} 3,3,5,5-$ Tetramethylbenzidine was added to $10 \mathrm{~mL}$ DI water to which $50 \mu \mathrm{L} 37 \% \mathrm{HCl}$ was added to dissolve the dye. $1 \mathrm{mg} / \mathrm{mL}$ Horseradish Peroxidase was dissolved in phosphate buffered saline (PBS) solution. A PMMA UV-vis cuvette was filled with $1 \mathrm{~mL} \mathrm{PBS,} 600 \mu \mathrm{L}$ of the dye solution, and $50 \mu \mathrm{L}$ of HRP solution. At 1, 8 and $22-$ minute intervals, $50 \mu \mathrm{L}$ aliquots of the reaction solution $(400 \mu \mathrm{L} 20.4 \mathrm{mg} / \mathrm{mL}$ GOx and $200 \mu \mathrm{L} 100 \mathrm{mM}$ glucose) was added to the cuvette and mixed thoroughly before collecting its spectrum using a Cary 6000i UVVis-NIR.

\section{TTF-TCNQ cathode preparation}

$80 \mu \mathrm{L}$ of a $40 \mathrm{mg} / \mathrm{mL}$ dispersion of TTF-TCNQ in acetonitrile was drop cast on an ITO/glass substrate and dried at ambient conditions overnight.

Two-electrode fuel cell measurements

The potential between the p-type material anode or TTF-TCNQ and ITO was measured by placing the working electrode on the p-type anode or TTF-TCNQ and shorting the counter and reference leads to the ITO electrode. The potential between $\mathrm{p}(\mathrm{gT} 2)$ and TTF-TCNQ was recorded by placing the working electrode on $\mathrm{p}(\mathrm{gT} 2)$ and shorting the counter and reference to TTF-TCNQ.

\section{OECT measurements}

Transfer and output characteristics of the OECTs were obtained using a custom LabVIEW program with a K2612 Keithley. Phosphate buffered saline solution was used as the electrolyte and Ag/AgCl pellet as gate.

\section{FC-OECT measurements}

The potential across the fuel cell was monitored using a 2-electrode setup on a Biologic SP-300 with the working electrode on the polymer and the reference/counter electrode on the ITO counter electrode. The $V_{\mathrm{D}}$ across the OECT was supplied by a K2612 Keithley and $I_{\mathrm{D}}$ measured using a custom LabVIEW program. ITO counter electrode was functionalized by drop casting $80 \mu \mathrm{L}$ of a $40 \mathrm{mg} / \mathrm{mL}$ dispersion of TTF-TCNQ in acetonitrile and dried at ambient conditions overnight. 


\section{H2: Supplementary Materials}

Fig. S1. Concentration change of $\mathrm{H}_{2} \mathrm{O}_{2}$ during glucose/GOx reaction.

Fig. S2. Chronoamperometry measurements of $\mathrm{p}(\mathrm{gT} 2)$ fuel cell anode current.

Fig. S3. Spectroelectrochemistry of $\mathrm{p}(\mathrm{gT} 2)$ in PBS.

Fig. S4. Characteristics of $\mathrm{p}(\mathrm{gT} 2)$ OECT.

Fig. S5. Performance of $\mathrm{p}(\mathrm{gT} 2) \mathrm{FC}-\mathrm{p}(\mathrm{gT} 2)$ in presence of different analyte concentrations.

Fig. S6. Application of $\mathrm{p}(\mathrm{gT} 2) \mathrm{FC}-\mathrm{p}(\mathrm{gT} 2)$ OECT to the oxidation of lactate and ethanol.

Fig. S7. Importance of gate current during gating of $\mathrm{p}(\mathrm{gT} 2)$ OECT.

Fig. S8. Cyclic Voltammetry of $\mathrm{p}(\mathrm{gT} 2)$.

Fig. S9. Volumetric charging of $\mathrm{p}(\mathrm{gT} 2)$.

Fig. S10. Glucose concentration dependence on fuel cell performance.

Fig. S11. Chronoamperometry of PEDOT:PSS.

Fig. S12. 3-electrode electrochemical characterization of TTF-TCNQ electron collector.

Fig. S13. Oxidation of N-type polymer p(NDI-T2)-90 in presence of glucose and GOx

Fig. S14. Selection of OECTs.

Table S1. Fuel Cell-OECT performance with p(gT2) OECT aspect ratio $W / L=200$ : Effect of Fuel Cell anode material.

Table S2. Fuel Cell-OECT performance: Effect of OECT Channel Material; $W / L=100$.

Table S3. Fuel Cell-OECT performance with p(gT2) on both fuel cell anode and OECT channel: Effect of OECT Channel Aspect Ratio.

Fig. S15. p(gT2) FC - p(gT2) OECT L20W2.

Fig. S16. $\mathrm{p}(\mathrm{gT} 2) \mathrm{FC}-\mathrm{p}(\mathrm{g} 2 \mathrm{~T}-\mathrm{TT})$ OECT L20W2.

Fig. S17. p(gT2) FC - PEDOT:PSS OECT L20W2.

Fig. S18. p(gT2) FC - p(gT2) OECT L10W2

Fig. S19. p(gPyDPP-MeOT2) FC - p(gT2) OECT L10W2.

\section{References and Notes}

1. D. Moia, A. Giovannitti, A. A. Szumska, I. P. Maria, E. Rezasoltani, M. Sachs, M. Schnurr, P. R. F.

Barnes, I. McCulloch, J. Nelson, Design and evaluation of conjugated polymers with polar side chains as electrode materials for electrochemical energy storage in aqueous electrolytes. Energy Environ. Sci., 1349-1357 (2019).

2. A. Giovannitti, D. T. Sbircea, S. Inal, C. B. Nielsen, E. Bandiello, D. A. Hanifi, M. Sessolo, G. G. Malliaras, I. McCulloch, J. Rivnay, Controlling the mode of operation of organic transistors through sidechain engineering. Proc. Natl. Acad. Sci. U. S. A. 113, 12017-12022 (2016).

3. J. Mei, Z. Bao, Side chain engineering in solution-processable conjugated polymers. Chem. Mater. 26, 604-615 (2014).

4. P. Romele, M. Ghittorelli, Z. M. Kovács-Vajna, F. Torricelli, Ion buffering and interface charge enable high performance electronics with organic electrochemical transistors. Nat. Commun. 10, 1-11 (2019).

5. D. Khodagholy, T. Doublet, P. Quilichini, M. Gurfinkel, P. Leleux, A. Ghestem, E. Ismailova, T. Hervé, S. Sanaur, C. Bernard, G. G. Malliaras, In vivo recordings of brain activity using organic transistors. Nat. Commun. 4 (2013), doi:10.1038/ncomms2573.

6. A. Michalska, A. Gałuszkiewicz, M. Ogonowska, M. Ocypa, K. Maksymiuk, PEDOT films: Multifunctional membranes for electrochemical ion sensing. J. Solid State Electrochem. 8, 381-389 (2004).

7. J. Gladisch, E. Stavrinidou, S. Ghosh, A. Giovannitti, M. Moser, I. Zozoulenko, I. Mcculloch, M. Berggren, Reversible Electronic Solid - Gel Switching of a Conjugated Polymer. 1901144 (2019), doi:10.1002/advs.201901144.

8. L. Torsi, M. Magliulo, K. Manoli, G. Palazzo, Organic field-effect transistor sensors: A tutorial review. Chem. Soc. Rev. 42, 8612-8628 (2013).

9. Y. Van De Burgt, E. Lubberman, E. J. Fuller, S. T. Keene, G. C. Faria, S. Agarwal, M. J. Marinella, A. Alec Talin, A. Salleo, A non-volatile organic electrochemical device as a low-voltage artificial synapse for neuromorphic computing. Nat. Mater. 16, 414-418 (2017). 
10. J. Rivnay, S. Inal, A. Salleo, R. M. Owens, M. Berggren, G. G. Malliaras, Organic electrochemical transistors. Nat. Rev. Mater. 3 (2018), doi:10.1038/natrevmats.2017.86.

11. D. A. Bernards, G. G. Malliaras, Steady-state and transient behavior of organic electrochemical transistors. Adv. Funct. Mater. 17, 3538-3544 (2007).

12. D. Khodagholy, J. Rivnay, M. Sessolo, M. Gurfinkel, P. Leleux, L. H. Jimison, E. Stavrinidou, T. Herve, S. Sanaur, R. M. Owens, G. G. Malliaras, High transconductance organic electrochemical transistors. Nat. Commun. 4, 1-6 (2013).

13. Q. Thiburce, A. Giovannitti, I. McCulloch, A. J. Campbell, Nanoscale Ion-Doped Polymer Transistors. Nano Lett. 19, 1712-1718 (2019).

14. L. Guan, L. Yu, G. Z. Chen, Capacitive and non-capacitive faradaic charge storage. Electrochim. Acta. 206, 464-478 (2016).

15. A. Giovannitti, R. B. Rashid, Q. Thiburce, B. D. Paulsen, C. Cendra, K. Thorley, D. Moia, J. T. Mefford, D. Hanifi, D. Weiyuan, M. Moser, A. Salleo, J. Nelson, I. McCulloch, J. Rivnay, Energetic Control of Redox-Active Polymers toward Safe Organic Bioelectronic Materials. Adv. Mater. 1908047, 1-9 (2020).

16. S. Inal, G. G. Malliaras, J. Rivnay, Benchmarking organic mixed conductors for transistors. Nat. Commun. 8, 1-6 (2017).

17. J. Rivnay, P. Leleux, M. Ferro, M. Sessolo, A. Williamson, D. A. Koutsouras, D. Khodagholy, M. Ramuz, X. Strakosas, R. M. Owens, C. Benar, J. M. Badier, C. Bernard, G. G. Malliaras, Highperformance transistors for bioelectronics through tuning of channel thickness. Sci. Adv. 1, 1-6 (2015).

18. W. Lee, D. Kim, J. Rivnay, N. Matsuhisa, T. Lonjaret, T. Yokota, H. Yawo, M. Sekino, G. G. Malliaras, T. Someya, Integration of Organic Electrochemical and Field-Effect Transistors for Ultraflexible, High Temporal Resolution Electrophysiology Arrays. Adv. Mater. 28, 9722-9728 (2016).

19. P. Leleux, J. Rivnay, T. Lonjaret, J. M. Badier, C. Bénar, T. Hervé, P. Chauvel, G. G. Malliaras, Organic electrochemical transistors for clinical applications. Adv. Healthc. Mater. 4, 142-147 (2015).

20. H. Sun, M. Vagin, S. Wang, X. Crispin, R. Forchheimer, M. Berggren, S. Fabiano, Complementary Logic Circuits Based on High-Performance n-Type Organic Electrochemical Transistors. Adv. Mater. 30, 1-7 (2018).

21. F. Pires, Q. Ferreira, C. A. V. Rodrigues, J. Morgado, F. C. Ferreira, Neural stem cell differentiation by electrical stimulation using a cross-linked PEDOT substrate: Expanding the use of biocompatible conjugated conductive polymers for neural tissue engineering. Biochim. Biophys. Acta - Gen. Subj. 1850, 1158-1168 (2015).

22. S. Stříteský, A. Marková, J. Víteček, E. Šafaříková, M. Hrabal, L. Kubáč, L. Kubala, M. Weiter, M. Vala, Printing inks of electroactive polymer PEDOT:PSS: The study of biocompatibility, stability, and electrical properties. J. Biomed. Mater. Res. - Part A. 106, 1121-1128 (2018).

23. W. Lee, D. Kim, N. Matsuhisa, M. Nagase, M. Sekino, G. G. Malliaras, T. Yokota, T. Someya, Transparent, conformable, active multielectrode array using organic electrochemical transistors. Proc. Natl. Acad. Sci. U. S. A. 114, 10554-10559 (2017).

24. D. A. Bernards, D. J. MacAya, M. Nikolou, J. A. Defranco, S. Takamatsu, G. G. Malliaras, Enzymatic sensing with organic electrochemical transistors. J. Mater. Chem. 18, 116-120 (2008).

25. S. Y. Yang, F. Cicoira, R. Byrne, F. Benito-Lopez, D. Diamond, R. M. Owens, G. G. Malliaras, Electrochemical transistors with ionic liquids for enzymatic sensing. Chem. Commun. 46, 7972-7974 (2010).

26. A. M. Pappa, D. Ohayon, A. Giovannitti, I. P. Maria, A. Savva, I. Uguz, J. Rivnay, I. McCulloch, R. M. Owens, S. Inal, Direct metabolite detection with an n-type accumulation mode organic electrochemical transistor. Sci. Adv. 4 (2018), doi:10.1126/sciadv.aat0911.

27. M. Braendlein, A.-M. Pappa, M. Ferro, A. Lopresti, C. Acquaviva, E. Mamessier, G. G. Malliaras, R. M. Owens, Lactate Detection in Tumor Cell Cultures Using Organic Transistor Circuits. Adv. Mater. 29, 1605744-1605750 (2017).

28. D. Ohayon, G. Nikiforidis, A. Savva, A. Giugni, S. Wustoni, T. Palanisamy, X. Chen, I. P. Maria, E. Di Fabrizio, P. M. F. J. Costa, I. Mcculloch, S. Inal, Biofuel powered glucose detection in bodily fluids with an n-type conjugated polymer. Nat. Mater. (2019), doi:10.1038/s41563-019-0556-4.

29. G. Cappon, M. Vettoretti, G. Sparacino, A. Facchinetti, Continuous glucose monitoring sensors for 
diabetes management: A review of technologies and applications. Diabetes Metab. J. 43, 383-397 (2019).

30. A. M. Pappa, V. F. Curto, M. Braendlein, X. Strakosas, M. J. Donahue, M. Fiocchi, G. G. Malliaras, R. M. Owens, Organic Transistor Arrays Integrated with Finger-Powered Microfluidics for Multianalyte Saliva Testing. Adv. Healthc. Mater. 5, 2295-2302 (2016).

31. H. Tang, P. Lin, H. L. W. Chan, F. Yan, Highly sensitive dopamine biosensors based on organic electrochemical transistors. Biosens. Bioelectron. 26, 4559-4563 (2011).

32. E. Macchia, R. A. Picca, K. Manoli, C. Di Franco, D. Blasi, L. Sarcina, N. Ditaranto, N. Cioffi, R. Österbacka, G. Scamarcio, F. Torricelli, L. Torsi, About the amplification factors in organic bioelectronic sensors. Mater. Horizons (2020), doi:10.1039/c9mh01544b.

33. R. A. S. Luz, A. R. Pereira, J. C. P. de Souza, F. C. P. F. Sales, F. N. Crespilho, Enzyme Biofuel Cells: Thermodynamics, Kinetics and Challenges in Applicability. ChemElectroChem. 1, 1751-1777 (2014).

34. R. Pauliukaite, A. Malinauskas, G. Zhylyak, U. E. Spichiger-Keller, Conductive organic complex salt TTF-TCNQ as a mediator for biosensors. An overview. Electroanalysis. 19, 2491-2498 (2007).

35. S. P. White, K. D. Dorfman, C. D. Frisbie, Label-free DNA sensing platform with low-voltage electrolyte-gated transistors. Anal. Chem. 87, 1861-1866 (2015).

36. D. W. Hwang, S. Lee, M. Seo, T. D. Chung, Recent advances in electrochemical non-enzymatic glucose sensors - A review. Anal. Chim. Acta. 1033, 1-34 (2018).

37. D. Josephy, T. Eling, R. Mason, The Horseradish Peroxidase-catalyzed Oxidation of 3,5,3',5'Tetramethylbenzidine. J. Biol. Chem. 257, 3669-3675 (1982).

38. C. Enengl, S. Enengl, S. Pluczyk, M. Havlicek, M. Lapkowski, H. Neugebauer, E. Ehrenfreund, DopingInduced Absorption Bands in P3HT: Polarons and Bipolarons. ChemPhysChem. 17, 3836-3844 (2016).

39. A. Giovannitti, I. P. Maria, D. Hanifi, M. J. Donahue, D. Bryant, K. J. Barth, B. E. Makdah, A. Savva, D. Moia, M. Zetek, P. R. F. Barnes, O. G. Reid, S. Inal, G. Rumbles, G. G. Malliaras, J. Nelson, J. Rivnay, I. McCulloch, The Role of the Side Chain on the Performance of N-type Conjugated Polymers in Aqueous Electrolytes. Chem. Mater. 30, 2945-2953 (2018).

40. J. Rivnay, P. Leleux, M. Sessolo, D. Khodagholy, T. Hervé, M. Fiocchi, G. G. Malliaras, Organic electrochemical transistors with maximum transconductance at zero gate bias. Adv. Mater. 25, 70107014 (2013).

41. H. Sun, J. Gerasimov, M. Berggren, S. Fabiano, N-Type organic electrochemical transistors: Materials and challenges. J. Mater. Chem. C. 6, 11778-11784 (2018).

42. A. Babel, S. A. Jenekhe, High Electron Mobility in Ladder Polymer Field-Effect Transistors. J. Am. Chem. Soc. 125, 13656-13657 (2003).

43. A. Onwubiko, W. Yue, C. Jellett, M. Xiao, H. Y. Chen, M. K. Ravva, D. A. Hanifi, A. C. Knall, B. Purushothaman, M. Nikolka, J. C. Flores, A. Salleo, J. L. Bredas, H. Sirringhaus, P. Hayoz, I. McCulloch, Fused electron deficient semiconducting polymers for air stable electron transport. Nat. Commun. 9, 1-9 (2018).

44. N. C. Foulds, C. R. Lowe, Enzyme Entrapment in Electrically Conducting Polymers. J. Chem. Soc. Faraday Trans. 1. 1, 1259-1264 (1986).

45. B. Oktay, S. Demir, N. Kayaman-Apohan, Preparation of a Poly(ethylene glycol)-Based Cross-Linked Network from a Click Reaction for Enzyme Immobilization. ChemistrySelect. 4, 6055-6059 (2019).

46. X. Chen, J. Shao, N. An, X. Li, H. Tian, C. Xu, Y. Ding, Self-powered flexible pressure sensors with vertically well-aligned piezoelectric nanowire arrays for monitoring vital signs. J. Mater. Chem. C. 3, 11806-11814 (2015).

47. O. Parlak, S. T. Keene, A. Marais, V. F. Curto, A. Salleo, Molecularly selective nanoporous membranebased wearable organic electrochemical device for noninvasive cortisol sensing. Sci. Adv. 4 (2018), doi:10.1126/sciadv.aar2904.

48. A. Giovannitti, C. B. Nielsen, D. T. Sbircea, S. Inal, M. Donahue, M. R. Niazi, D. A. Hanifi, A. Amassian, G. G. Malliaras, J. Rivnay, I. McCulloch, N-type organic electrochemical transistors with stability in water. Nat. Commun. 7, 1-9 (2016). 


\section{Acknowledgments}

General: We thank the Dionne lab for access to their UV-vis spectrometer and the Soft and Hybrid Materials Facility in the Stanford Nano Shared Facilities for access to the rheometer and profilometer.

Funding: A.G. and A.S. acknowledge funding from the TomKat Center for Sustainable Energy at Stanford University. A.S. and M.T. gratefully acknowledge support from the National Science Foundation Award CBET \#1804915. A.M. gratefully acknowledges support from the Knut and Alice Wallenberg Foundation (KAW 2016.0494) for Postdoctoral Research at Stanford University. Part of this work was performed at the Stanford Nano Shared Facilities (SNSF), supported by the National Science Foundation under award ECCS-1542152.

Author contributions: M.T., A.G., A.M. and A.S. conceived of the project idea. M.T. and A.G. designed and executed experiments for the paper. A.M. fabricated patterned dies used for OECTs. M.T., A.G. and A.S. analyzed the data and wrote the manuscript. M.M. synthesized polymers utilized in the study. B.C. designed the setup for spectroelectrochemical measurements. D.S. helped with the design of spectroelectrochemical measurements.

Competing interests: The authors declare that there are no competing interests.

Data and materials availability: All data needed to evaluate the conclusions in the paper are present in the paper and/or the Supplementary Materials. Additional data related to this paper may be requested from the authors. 
A
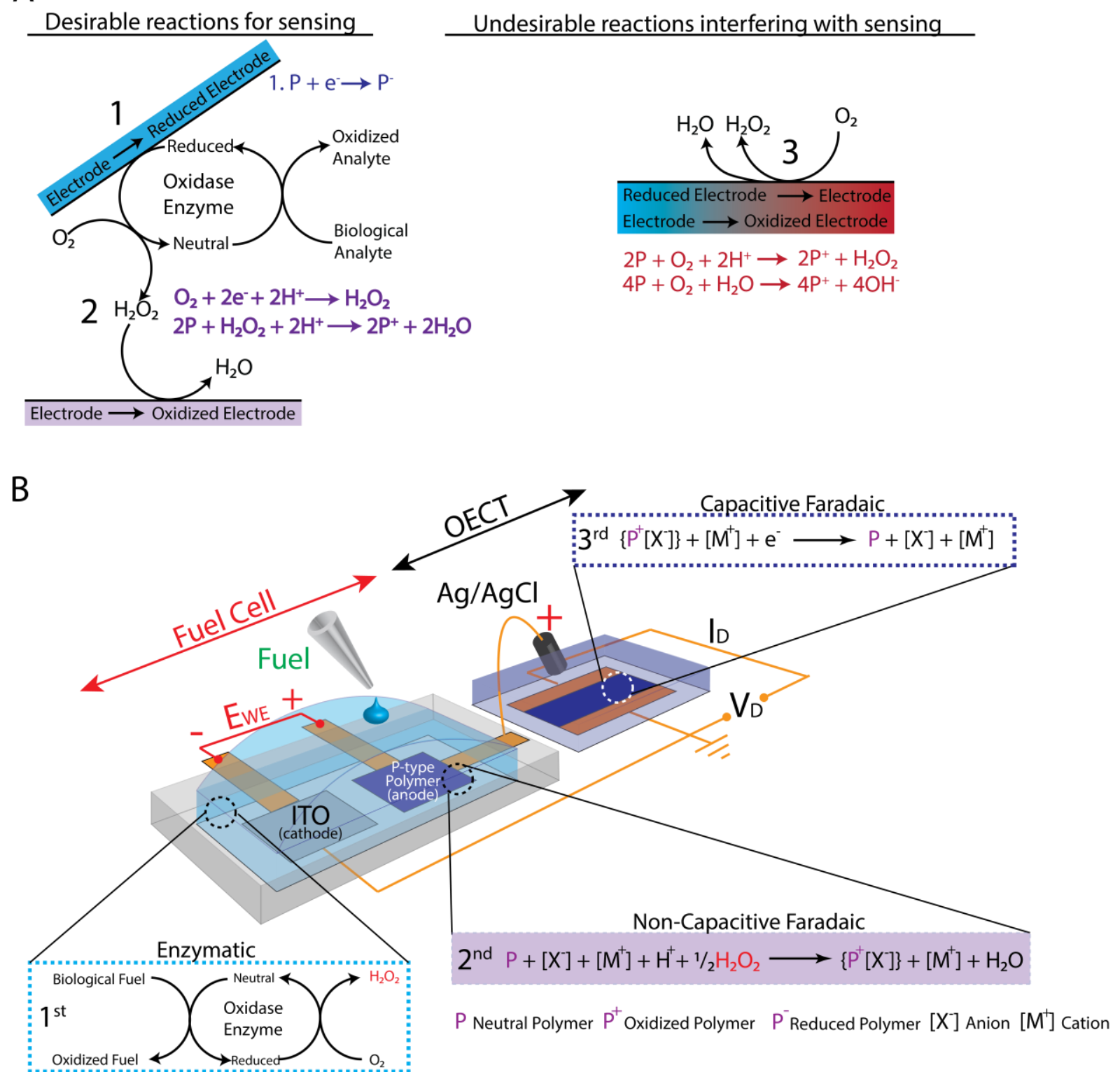

Fig. 1. Electrochemical reactions and operation of Fuel Cell OECT. (A) Reaction scheme showing chemical and electrocehmical reactions that can occur between redox-active materials and the analyte/enzyme solution in the presence of $\mathrm{O}_{2}$. Reaction 1: Direct electron transfer (DET) from the enzyme to the OMIEC. Reaction 2: Indirect electron transfer (IET) from the enzyme to ambient $\mathrm{O}_{2}$ to form $\mathrm{H}_{2} \mathrm{O}_{2}$ (reactive intermediate) that acts as an oxidant to charge the OMIEC. Reaction 3: Undesirable side reactions: Oxygen reduction reactions (ORR) of the OMIEC by molecular oxygen form $\mathrm{H}_{2} \mathrm{O}_{2}$ (two-electron process) or $\mathrm{H}_{2} \mathrm{O}$ (four-electron process). (B) Chemical oxidation reaction of the OMIEC is performed solely on the fuel cell, which leads to the charging of the fuel cell anode that generates a potential to drive electrochemical reactions on the gate electrode ( $\mathrm{Ag} / \mathrm{AgCl}$ pellet electrode) of the OECT. Potential across the p-type polymer (anode) and ITO counter electrode (cathode) of the fuel cell (potential (working electrode) $E_{\mathrm{WE}}$ ) and OECT source-drain current $\left(I_{\mathrm{D}}\right)$ are measured simultaneously. Device operation: (1) Intentional generation of $\mathrm{H}_{2} \mathrm{O}_{2}$ by enzymatic reactions (glucose and GOx) in the electrolyte of the fuel cell, (2) chemical oxidation (non-capacitive faradaic reactions) of $\mathrm{p}$-type polymer on the fuel cell anode by $\mathrm{H}_{2} \mathrm{O}_{2}$ leading to increase in potential, (3) that result in electrochemical redox-reactions at the OECT gate electrode and thus charging/discharging of the p-type polymer in OECT channel (capacitive faradaic reduction). 

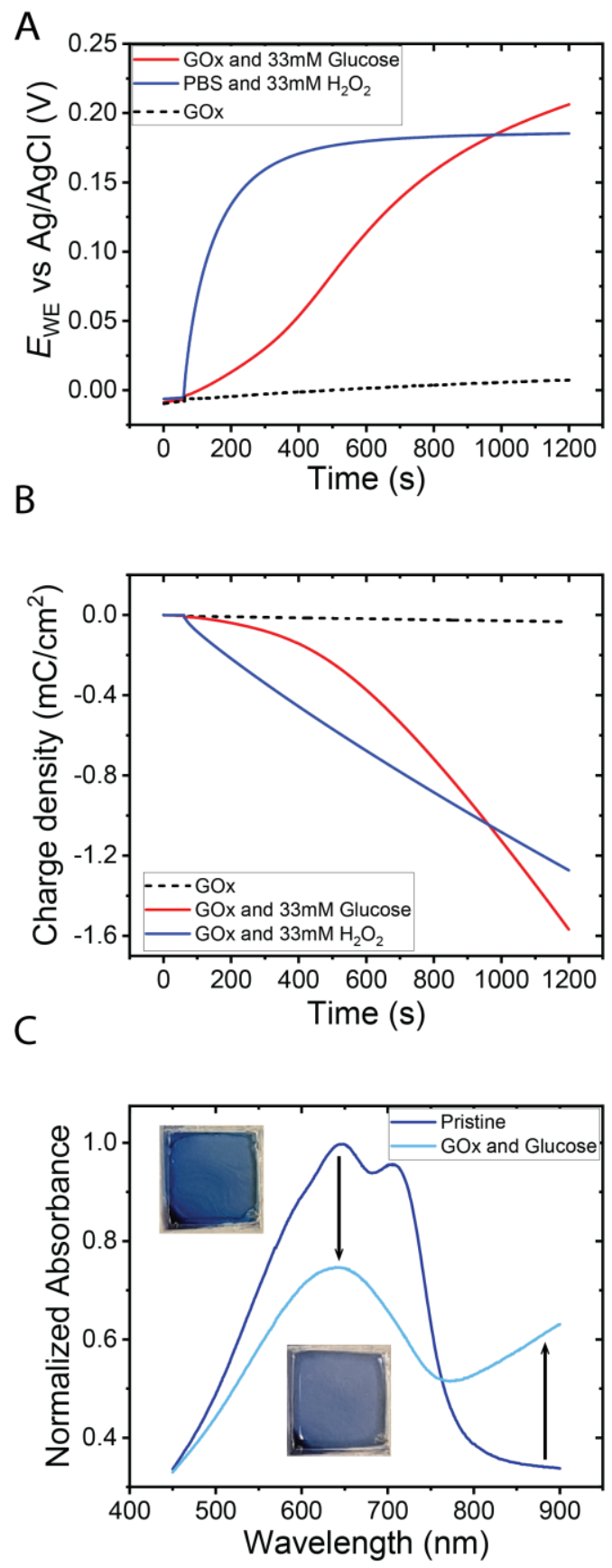

Fig. 2. Oxidation mechanism of fuel cell anode p(gT2). (A) Monitoring of the open circuit voltage (OCV) of the anode during the enzymatic reaction ( $33 \mathrm{mM}$ glucose in presence of GOx) (red) and after the addition of 33 $\mathrm{mM} \mathrm{H} \mathrm{O}_{2}$ to PBS (blue) for 20 minutes. (B) Chronoamperometry measurements during a similar series of experiments conducted in Fig. 2A. A fixed potential corresponding to its initial OCV in absence of reactants $(0$ $\mathrm{V}$ vs. sat $\mathrm{Ag} / \mathrm{AgCl})$ was applied on $\mathrm{p}(\mathrm{gT} 2)$ to measure the charge injected into $\mathrm{p}(\mathrm{gT} 2)$ to prevent oxidation of the polymer due to HPRR. (C) Spectrochemical measurements of p(gT2) by monitoring changes of its absorption spectrum during the oxidation after performing the enzymatic reactions with $33 \mathrm{mM}$ glucose in presence of GOx. Absorbance (scaled to the peak of pristine film at $\lambda_{\max }=650 \mathrm{~nm}$ ) of neat polymer (dark blue) and after reaction with glucose and GOx (light blue). Arrows indicate changes in intensity of HOMO-LUMO and polaron transitions. Insets show color change of film before and after the reaction, showing the bleaching of film color. Note that due to side reactions with oxygen in ambient, the neat polymer is initially slightly charged (see spectroelectrochemical characteristics in Fig. S5). 
A

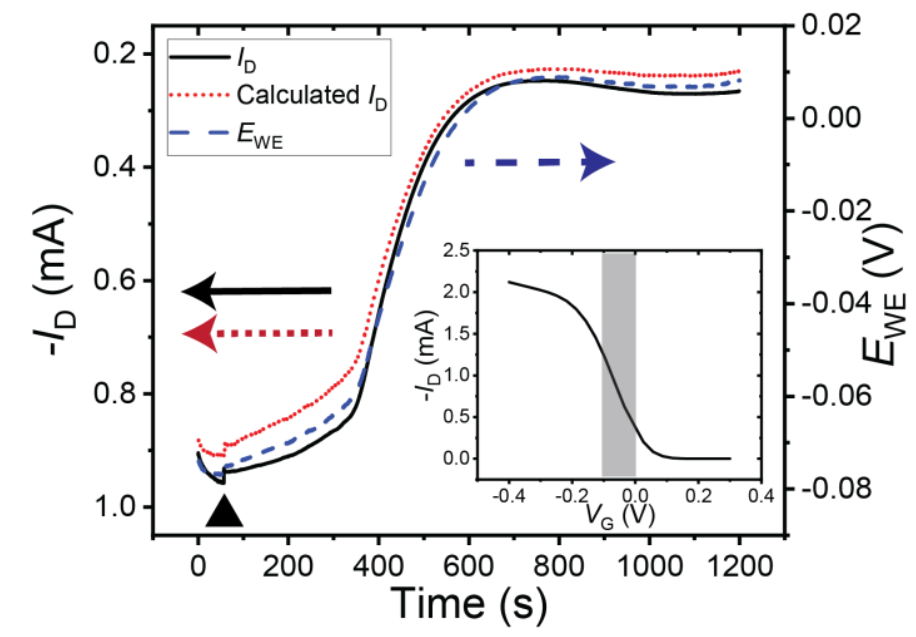

B

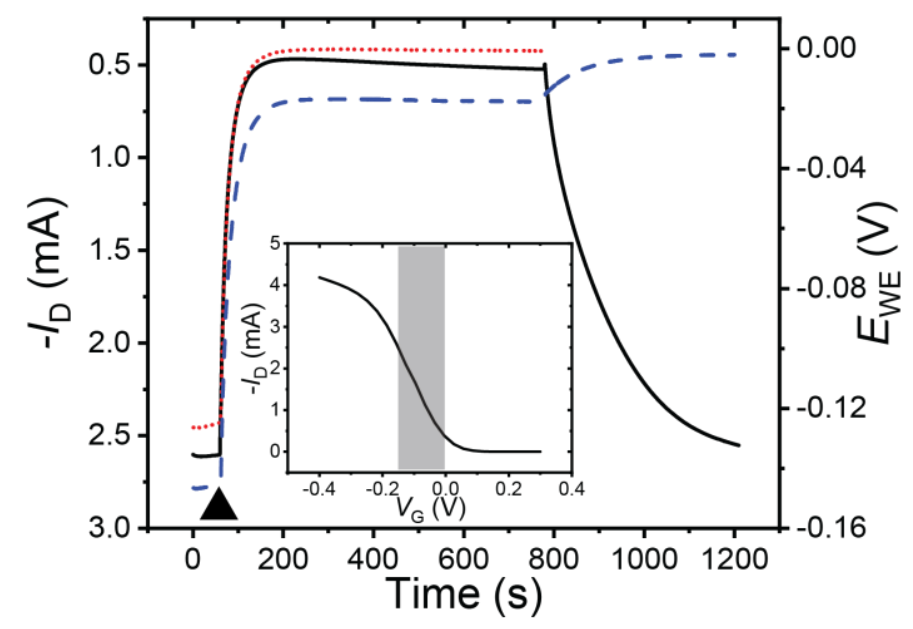

Fig. 3. Performance of Fuel Cell OECTs using p(gT2) FC - p(gT2) OECT. (A) FC-OECT operated by glucose/GOx. The OECT is operated in PBS solution with $V_{\mathrm{D}}=-0.1 \mathrm{~V}$ with OECT dimensions of $L=10 \mu \mathrm{m} ; W=2000 \mu \mathrm{m} ; \mathrm{d}=120 \mathrm{~nm}$. The electrolyte for the FC is GOx in PBS aqueous electrolyte at the beginning of the experiment, and glucose $(33 \mathrm{mM})$ added after $60 \mathrm{~s}$ (indicated by the triangle). The potential change across fuel cell $\left(E_{\mathrm{WE}}\right)$ is shown in blue dashed line, $I_{\mathrm{D}}$ in black and the calculated $I_{\mathrm{D}}$ based on OECT transfer curve is depicted as a red dotted line. The inset shows the transfer curve with $V_{\mathrm{D}}=-0.1 \mathrm{~V}$ (the grey rectangle highlights the oxidation window range of the FC electrode). (B) $\mathrm{FC}-\mathrm{OECT}$ control experiment with $33 \mathrm{mM} \mathrm{H}_{2} \mathrm{O}_{2}$ added to $\mathrm{PBS}$, using $V_{\mathrm{D}}=-0.2 \mathrm{~V}$ and the same OECT channel dimensions as shown in (A). The triangle indicates the time when the $\mathrm{H}_{2} \mathrm{O}_{2}$ solution is added to PBS in the FC compartment. Fuel cell was physically disconnected from the OECT at $780 \mathrm{~s}$. Note that a different $V_{\mathrm{D}}$ is utilized for (B) to demonstrate that $I_{\mathrm{D}}$ can be scaled with $V_{\mathrm{D}}$. Refer to Tables S1-3 for more detailed comparison of FC-OECT operation for glucose and $\mathrm{H}_{2} \mathrm{O}_{2}$ fuels at varied $V_{D}$. 


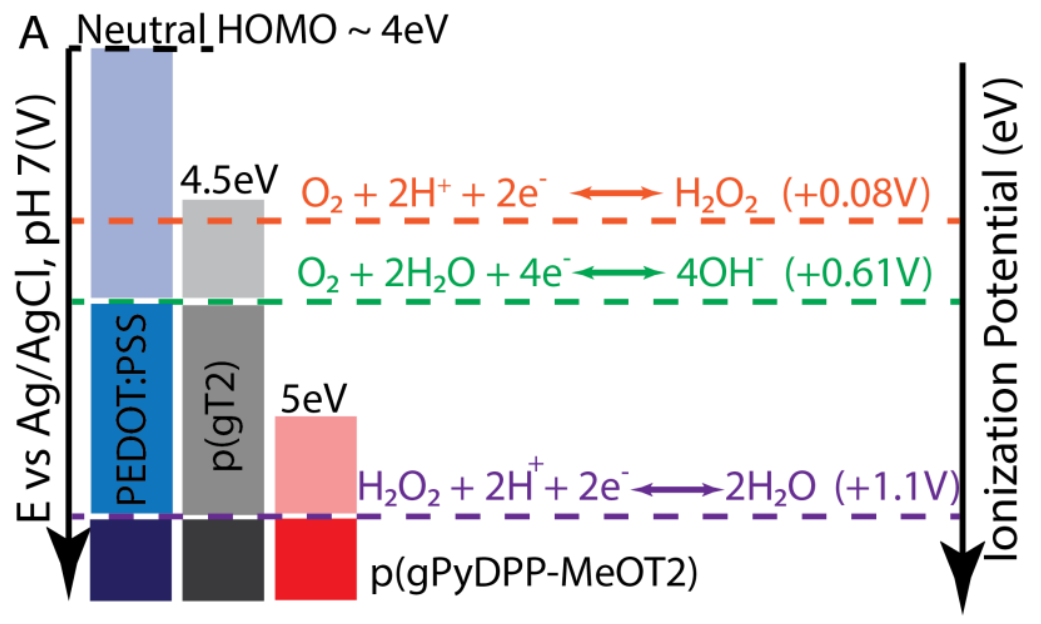

B

C

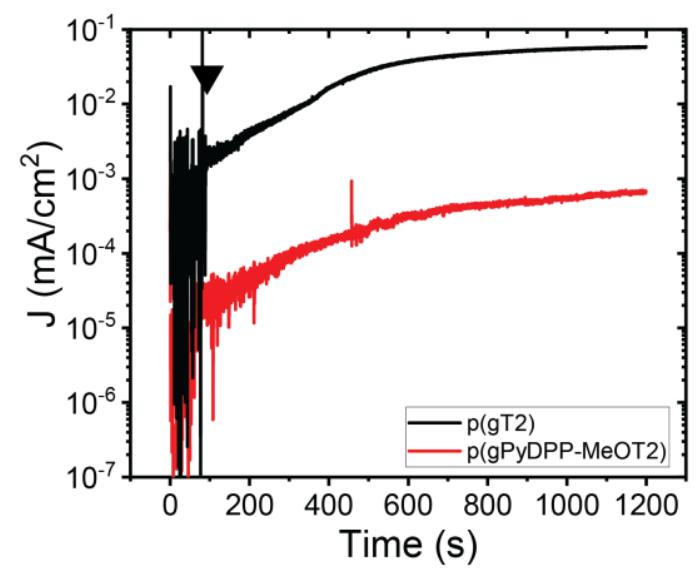

D

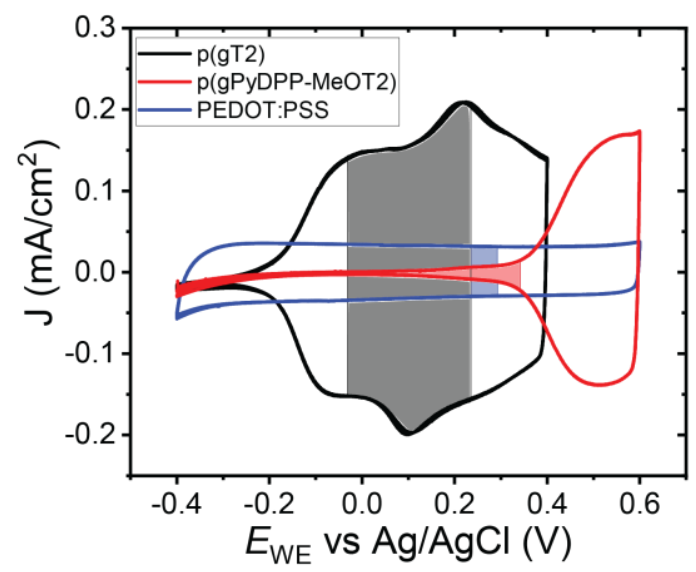

Fig. 4. Choice of anode material for the fuel cell. (A) Schematic of the energy levels of the ptype polymers PEDOT:PSS (blue), p(gT2) (black) and p(gPyDPP-MeOT2) (red) relative to ORR and HPRR. Lighter colors indicate that the polymers are charged (oxidized) due to ORR (ambient conditions). (B) OCV measurements of thin films of the polymer series on ITO in GOx/PBS electrolyte. Triangle indicates the addition of glucose $(33 \mathrm{mM})$. (C) CA measurements of thin films of $\mathrm{p}(\mathrm{gT} 2)(0 \mathrm{~V}$ vs. $\mathrm{Ag} / \mathrm{AgCl})$ and $\mathrm{p}(\mathrm{gPyDPP}-\mathrm{MeOT} 2)(+0.07 \mathrm{~V}$ vs. $\mathrm{Ag} / \mathrm{AgCl})$ on ITO with $33 \mathrm{mM}$ glucose in presence of GOx. Triangle indicates the addition of glucose. (D) CV measurements of the polymer series in PBS solution with glucose $(33 \mathrm{mM}) / \mathrm{GOx}$ solution at $50 \mathrm{mV} / \mathrm{s}$. The highlighted area of the CV curves indicates the corresponding potential change of the polymers after being immersed into the electrolytes containing $33 \mathrm{mM}$ glucose and GOx from OCV measurements shown in (B). 
A

B
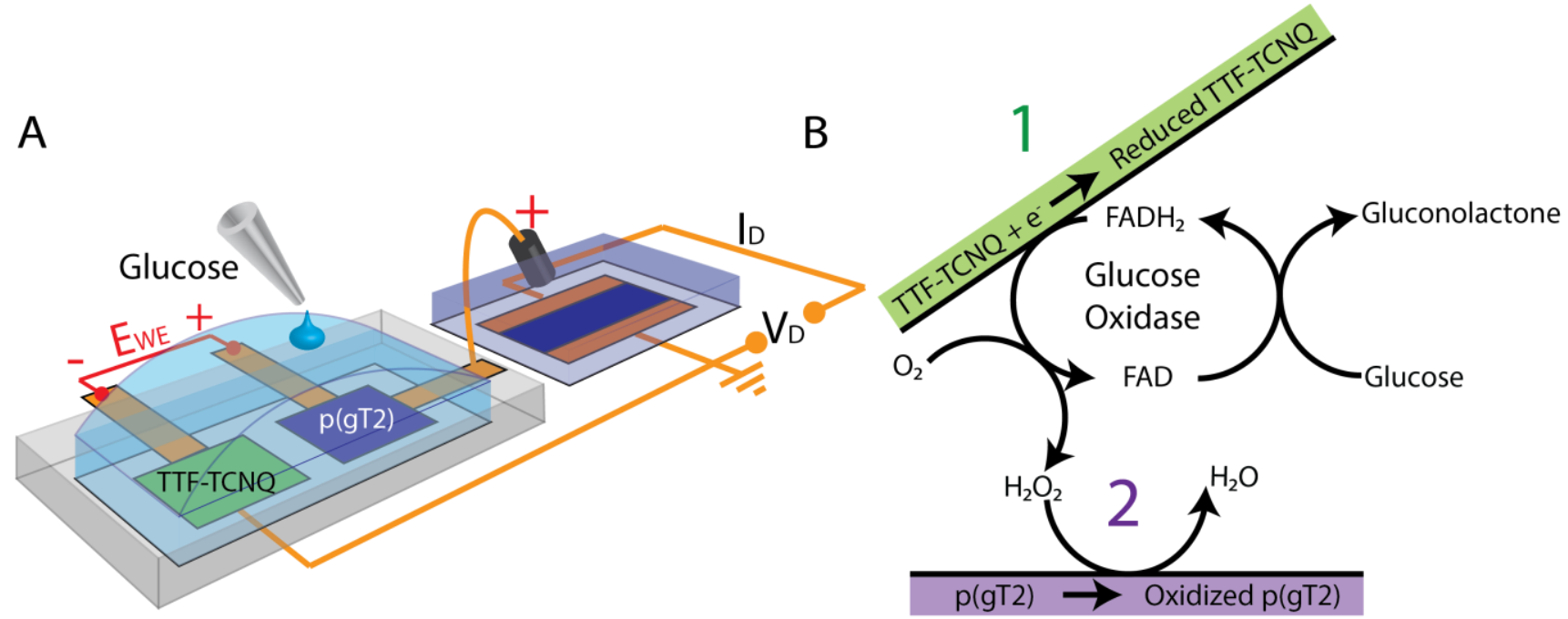

C

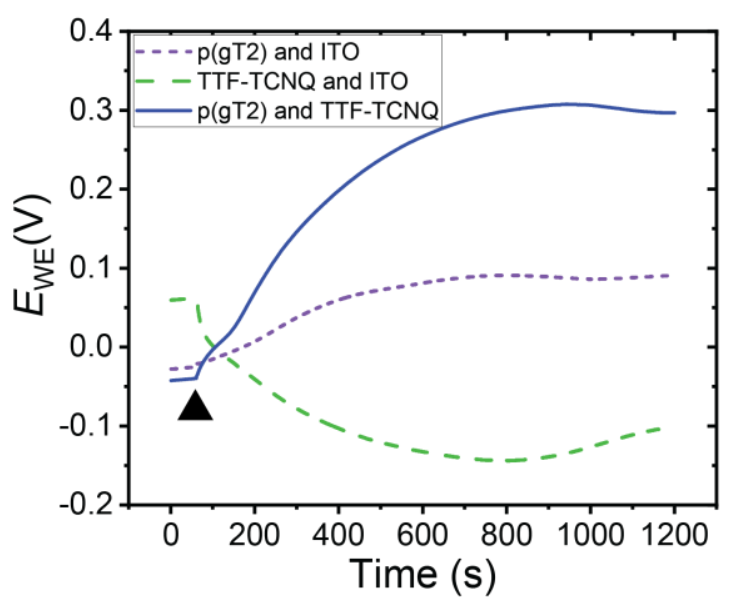

D

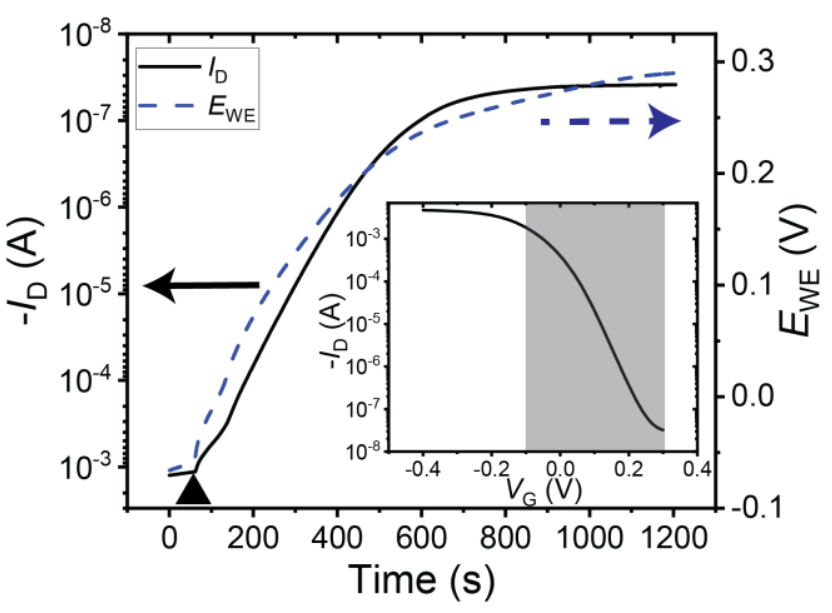

Fig. 5. Optimization of the FC: Functionalization of the FC cathode with the electron acceptor TTF-TCNQ to improve FC-OECT performance. (A) Schematic of FC-OECT with functionalization of the cathode with TTF-TCNQ. (B) Reaction scheme showing (1) reduction of TTF-TCNQ via direct electron transfer from GOx and (2) oxidation of $\mathrm{p}(\mathrm{gT} 2)$ by $\mathrm{H}_{2} \mathrm{O}_{2}$ (C) OCV measurements of the FC (2-electrode measurements) for thin films of $\mathrm{p}(\mathrm{gT} 2)$ (blue line), TTFTCNQ (green dashed line) and a combination of $\mathrm{p}(\mathrm{gT} 2)$ and TTF-TCNQ (dashed purple line), after the addition of glucose $(33 \mathrm{mM})$ to GOx in PBS. (D) Performance of the FC-OECT with a TTFTCNQ-p(gT2) FC and p(gT2)-OECT $\left(V_{\mathrm{D}}=-0.2 \mathrm{~V}, I_{\mathrm{D}}\right.$ (black) and $E_{\mathrm{WE}}$ (blue dashed)) after the addition of $33 \mathrm{mM}$ glucose to GOx in PBS. Inset shows the transfer curve $\left(V_{\mathrm{D}}=-0.2 \mathrm{~V}\right.$, the grey rectangle highlights the potential shift of the FC electrode during reaction). 


\section{Supplementary Materials}

\section{$\underline{\text { Summary of enzymatic reactions }}$}

1) Oxidation of glucose by Glucose Oxidase

2) Oxidation of lactate by Lactate Oxidase

$$
\text { Glucose }+\mathrm{O}_{2} \rightarrow \text { Gluconolactone }+\mathrm{H}_{2} \mathrm{O}_{2}
$$

3) Oxidation of ethanol by Alcohol Oxidase

$$
\text { Lactate }+\mathrm{O}_{2} \rightarrow \text { Pyruvate }+\mathrm{H}_{2} \mathrm{O}_{2}
$$

$$
\text { Ethanol }+\mathrm{O}_{2} \rightarrow \text { Acetaldehyde }+\mathrm{H}_{2} \mathrm{O}_{2}
$$

$\underline{\text { Verification of } \mathrm{H}_{2} \underline{\mathrm{O}}_{2} \text { consumption by } \mathrm{p}(\mathrm{gT} 2)}$

The formation of $\mathrm{H}_{2} \mathrm{O}_{2}$ by glucose/GOx was verified using a method for $\mathrm{H}_{2} \mathrm{O}_{2}$ detection based on Horseradish Peroxidase oxidation of the dye 3,3',5,5'-Tetramethylbenzidine (37) and UV-vis spectroscopy, where the absorbance of the oxidized dye is proportional to the concentration of $\mathrm{H}_{2} \mathrm{O}_{2}$ in solution. We monitor the formation of $\mathrm{H}_{2} \mathrm{O}_{2}$ formation and changes after the electrolyte was in contact with a thin film of $\mathrm{p}(\mathrm{gT} 2)$. As expected, the concentration of $\mathrm{H}_{2} \mathrm{O}_{2}$ in the reaction solution is lower in the presence of polymer $\mathrm{p}$ (gT2) (red lines).

(a)

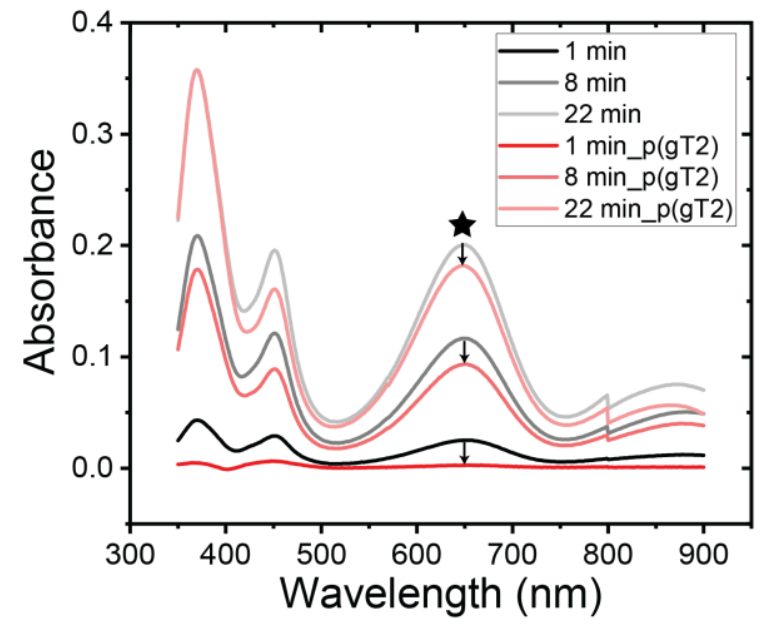

(b)

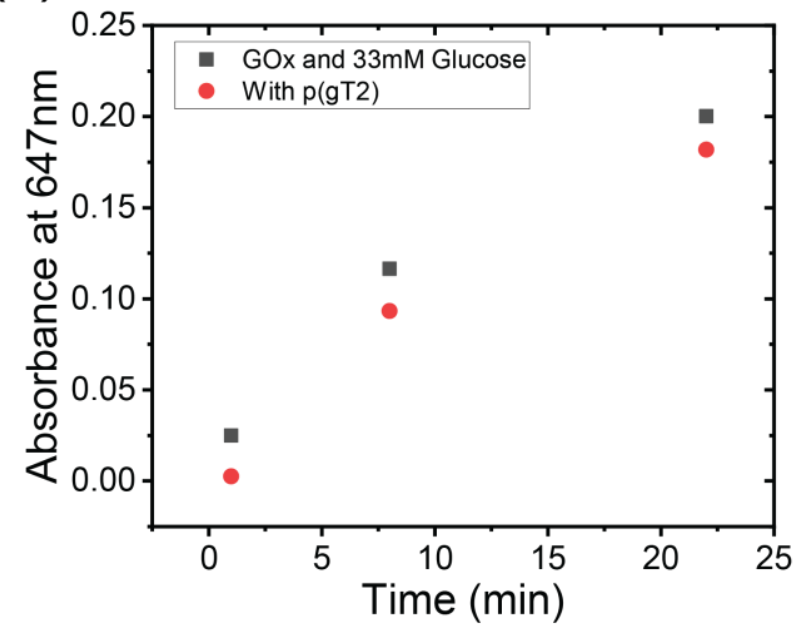

Figure S1. (a) UV Vis absorption spectrum for 3,3',5,5'-tetramethylbenzidine and Horseradish Peroxidase during glucose/GOx reaction and changes in absorption spectrum over time due to $\mathrm{H}_{2} \mathrm{O}_{2}$ formation. Aliquots of the electrolyte containing glucose/GOx are taken at different time intervals (1, 8 and $22 \mathrm{~min}$ ) in absence (grey lines) and presence (red lines) of $\mathrm{p}(\mathrm{gT} 2)$. (b) Change in absorbance of the dye (at $647 \mathrm{~nm}$ ) over time in absence (black) and presence (red) of a p(gT2) thin film on ITO. 


\section{Effect of mass transport on rate of oxidation of $\mathrm{p}(\mathrm{gT} 2) \mathrm{FC}$}

The ease of mass transport within the reaction solution is another important factor in FC performance. The rate of potential change (oxidation of $\mathrm{p}(\mathrm{gT} 2)$ ) across the FC was found to be dependent on the concentration of glucose where the highest rates for the oxidation were found for a solution with a concentration of $33 \mathrm{mM}$ glucose. One would expect the rate of formation of $\mathrm{H}_{2} \mathrm{O}_{2}$ and hence the rate of oxidation of $\mathrm{p}(\mathrm{gT} 2)$ to be proportional to the concentration of glucose in solution, according to the first-order reaction kinetics of GOx. However, increasing the glucose concentration to higher concentrations increases the viscosity of the glucose solutions, likely limiting mass transport of glucose and/or $\mathrm{H}_{2} \mathrm{O}_{2}$, resulting in a decrease in the rate of $\mathrm{p}(\mathrm{gT} 2)$ oxidation. $33 \mathrm{mM}$ glucose gave the largest change in potential of $\mathrm{p}(\mathrm{gT} 2)$. Hence, this concentration was used in subsequent experiments.

(a)

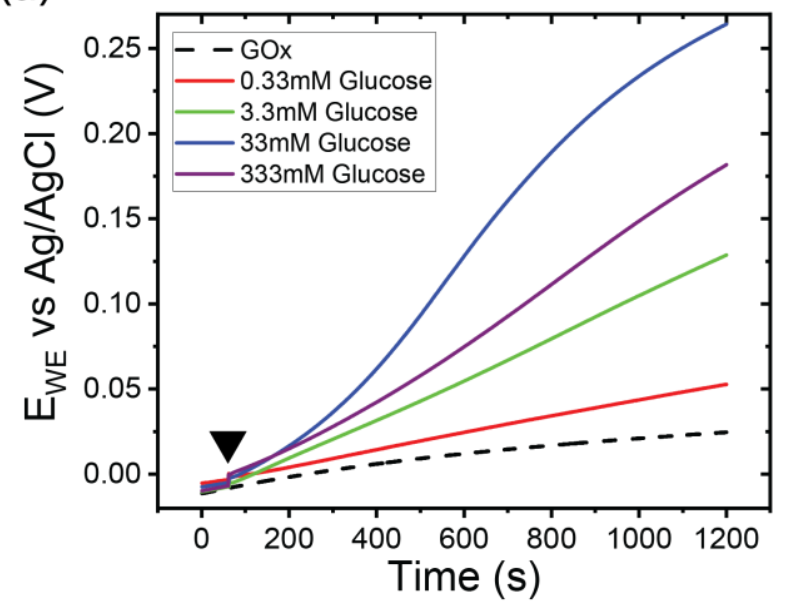

(b)

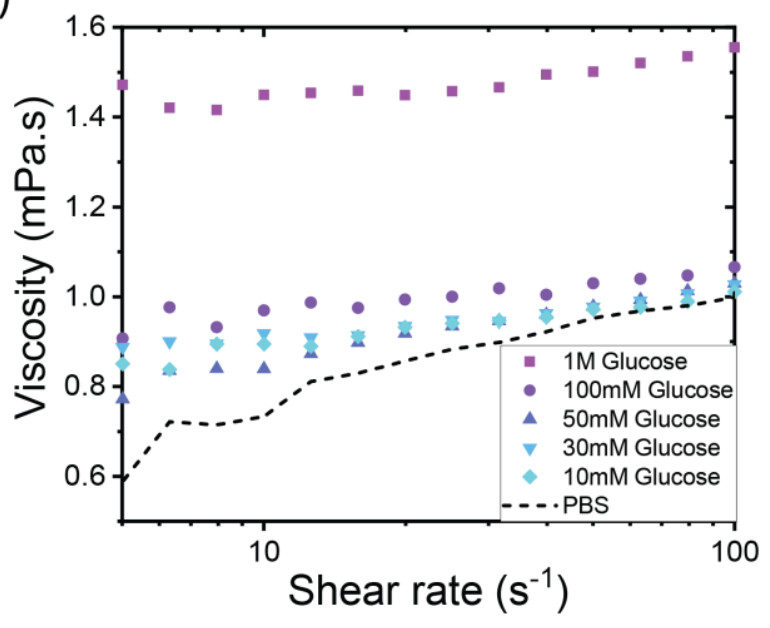

Figure S2. (a) OCV measurement of thin films of $\mathrm{p}(\mathrm{gT} 2)$ with various concentrations of glucose (in PBS). (b) Viscosity measurements for various glucose concentrations. 


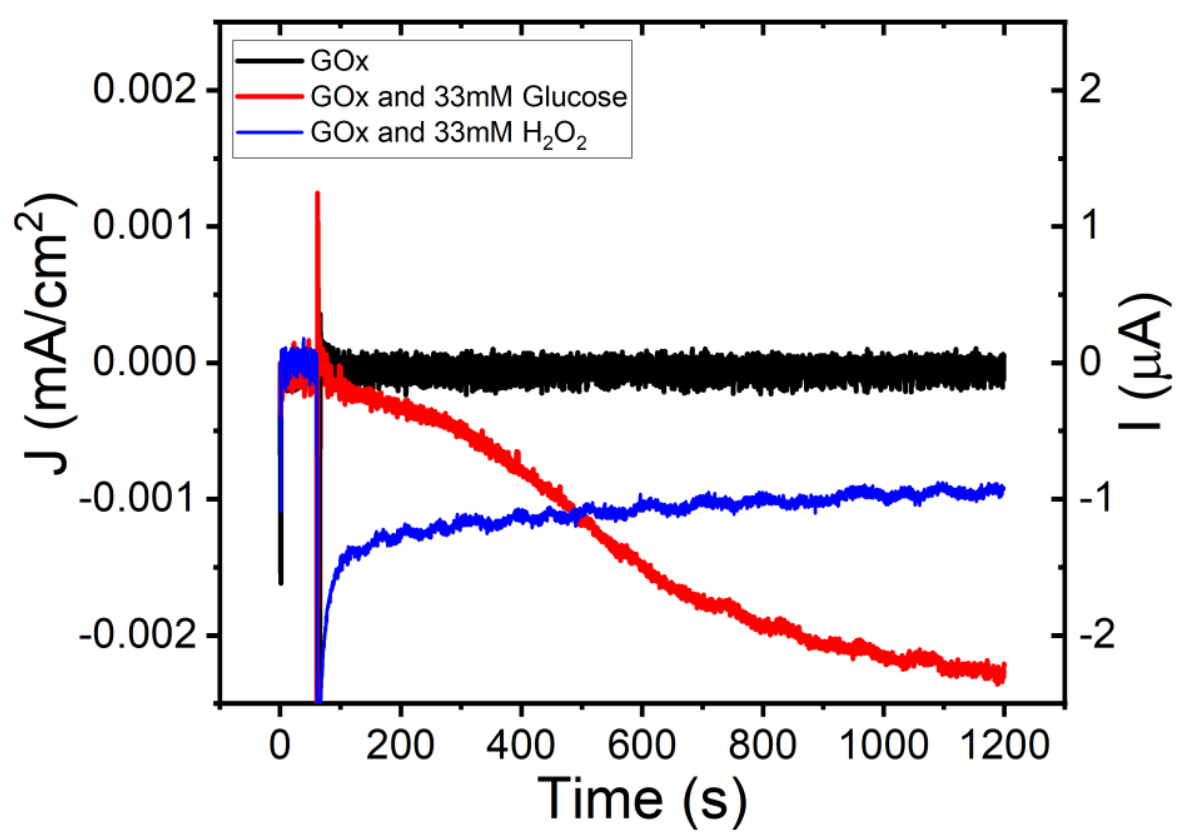

Figure S3. CA measurements of thin films of $\mathrm{p}(\mathrm{gT} 2)$ anode on ITO. Control experiment PBS and GOx solution (black), PBS, GOx and $33 \mathrm{mM}$ glucose (red) and PBS and $33 \mathrm{mM} \mathrm{H}_{2} \mathrm{O}_{2}$ (blue).

Cyclic Voltammetry measurements of $\mathrm{p}(\mathrm{gT} 2)$

The cyclic-voltammogram of $\mathrm{p}(\mathrm{gT} 2)$ in PBS (pH neutral) is shown in Fig. S4. Adding glucose to a solution of GOx results in redox activity $<0 \mathrm{~V}$ vs $\mathrm{Ag} / \mathrm{AgCl}$ which can be attributed to the reduction of $\mathrm{H}_{2} \mathrm{O}_{2}$ that formed during the enzymatic reaction (green). A similar yet larger magnitude of redox activity was observed in controls with equivalent concentrations of $\mathrm{H}_{2} \mathrm{O}_{2}$ (red dashed). Replacing the electrolyte with fresh PBS solution after each reaction recovers the redox characteristics of $\mathrm{p}(\mathrm{gT} 2)$ that are comparable to its pristine state. This further indicates the formation of $\mathrm{H}_{2} \mathrm{O}_{2}$ by the enzymatic reaction and that the chemical redox reactions on $\mathrm{p}(\mathrm{gT} 2)$ are reversible.

(a)

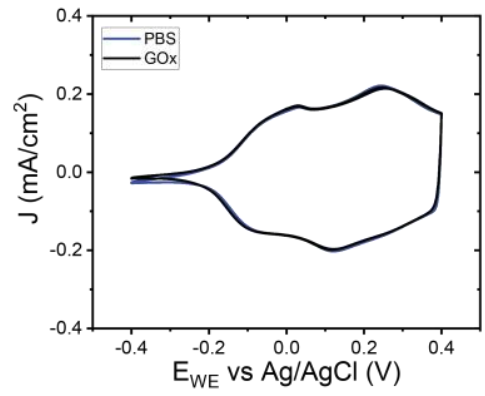

(b)

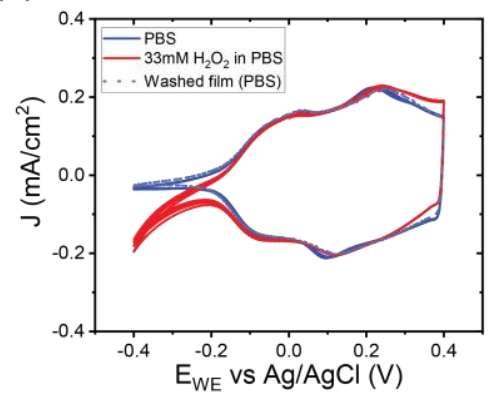

(c)

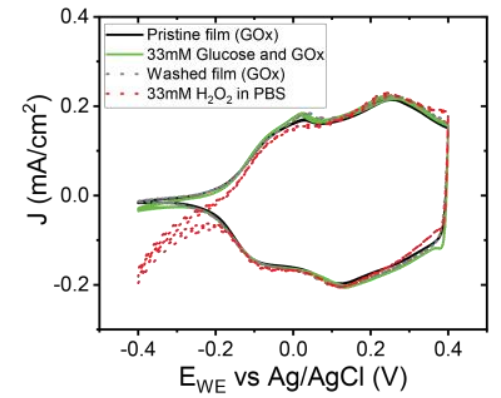

Figure S4. CV measurements of thin films of $\mathrm{p}(\mathrm{gT} 2)$ on ITO with a scan rate of $50 \mathrm{mV} / \mathrm{s}$ in ambient conditions in (a) neat PBS (blue) and after the addition of GOx (black), (b) addition of $\mathrm{H}_{2} \mathrm{O}_{2}$ (33 $\mathrm{mM}$ ) (red), followed by washing of the fuel cell anode with PBS (blue dotted) (c) $33 \mathrm{mM}$ glucose in presence of GOx in PBS (green), followed by washing of the fuel cell anode with PBS and addition of GOx (black dotted), for comparison, the $\mathrm{CV}$ of the polymer in $33 \mathrm{mM} \mathrm{H} \mathrm{H}_{2} \mathrm{O}_{2}$ is shown (red dotted). 


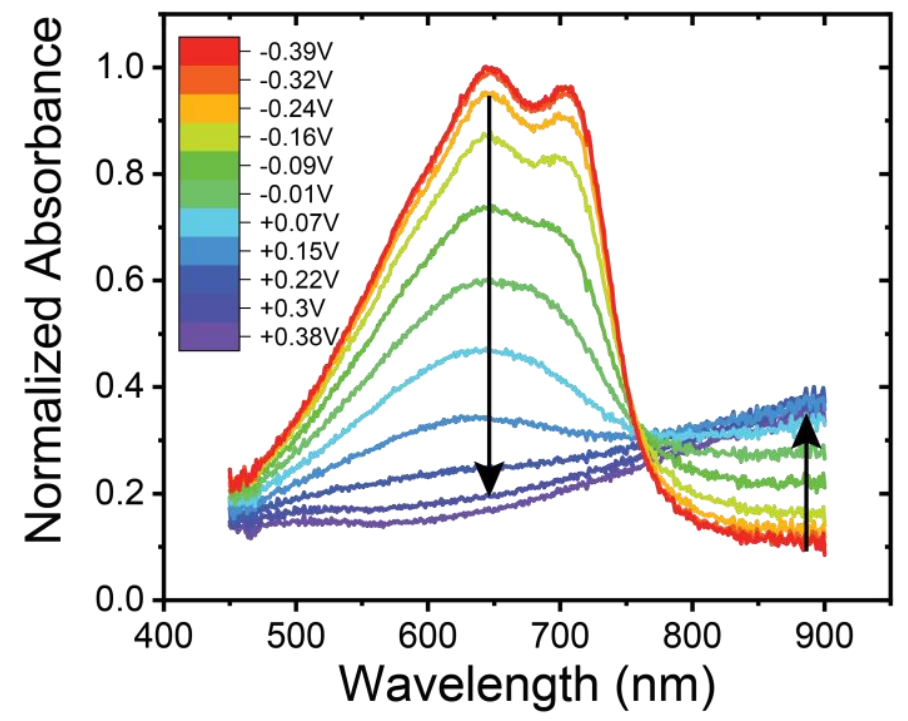

Figure S5. Spectroelectrochemistry of $\mathrm{p}(\mathrm{gT} 2)$ on ITO in PBS in ambient conditions using a threeelectrode setup for CV measurements (scan rate of $50 \mathrm{mV} / \mathrm{s}$ ) shown in Figure S4a. The potentials are reported vs. $\mathrm{Ag} / \mathrm{AgCl}$ and the results are in agreement with findings in the literature (1)

\section{$\mathrm{p}(\mathrm{gT} 2) \mathrm{FC}-\mathrm{p}(\mathrm{gT} 2) \mathrm{OECT}$}

(a)

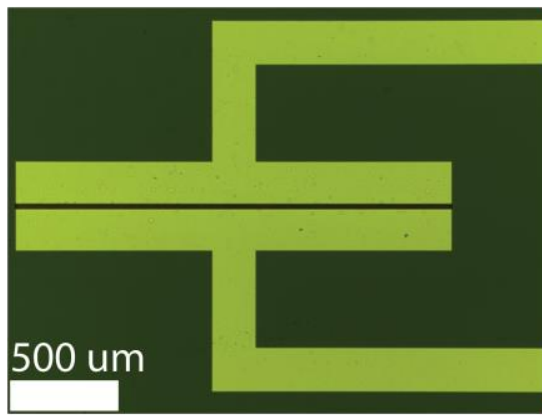

(c)

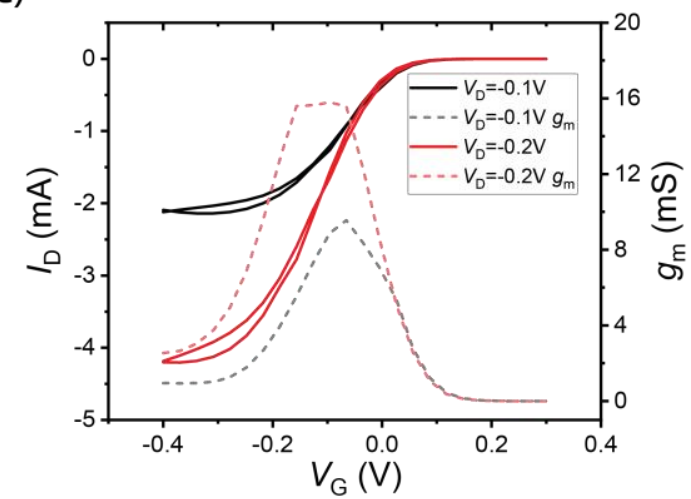

(b)

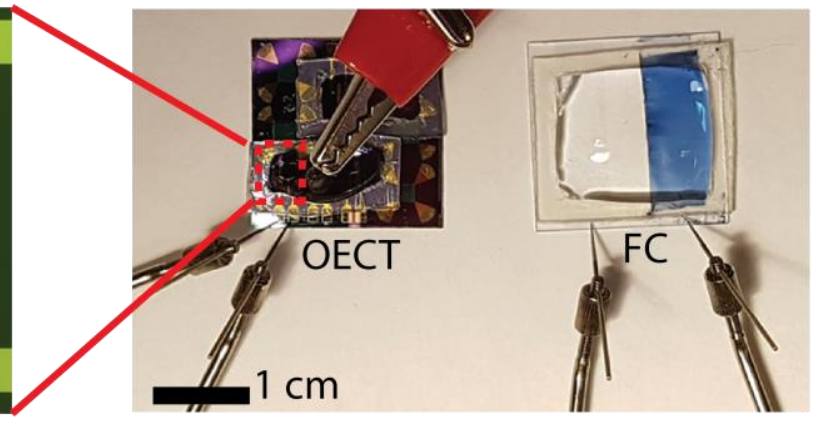

(d)

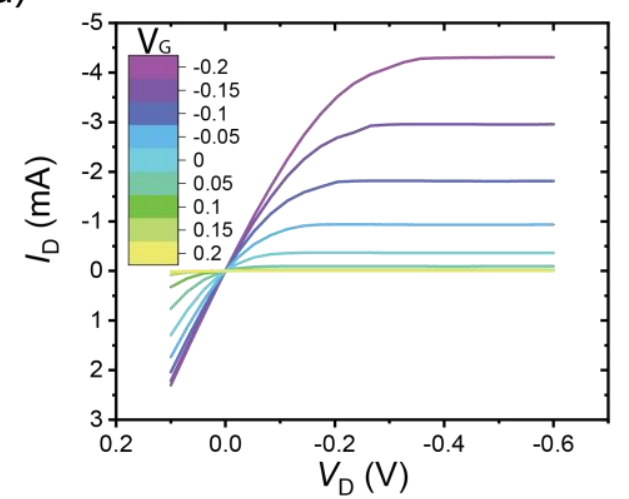

Figure S6. Characteristics of $\mathrm{p}(\mathrm{gT} 2)$ OECT and FC-OECT. (a) Micrograph of the OECT channel with dimensions of $W=2 \mathrm{~mm}, L=10 \mu \mathrm{m}$. (b) Image of the FC-OECT during operation of the device. (c) OECT transfer curves (solid lines) and transconductance (dashed lines) at $\mathrm{V}_{\mathrm{D}}=-0.1 \mathrm{~V}$ (black) and $\mathrm{V}_{\mathrm{D}}=-0.2 \mathrm{~V}$ (red) using PBS as the electrolyte. (d) Output curves of p(gT2) OECT 


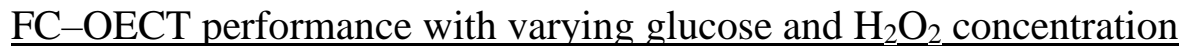

(a)

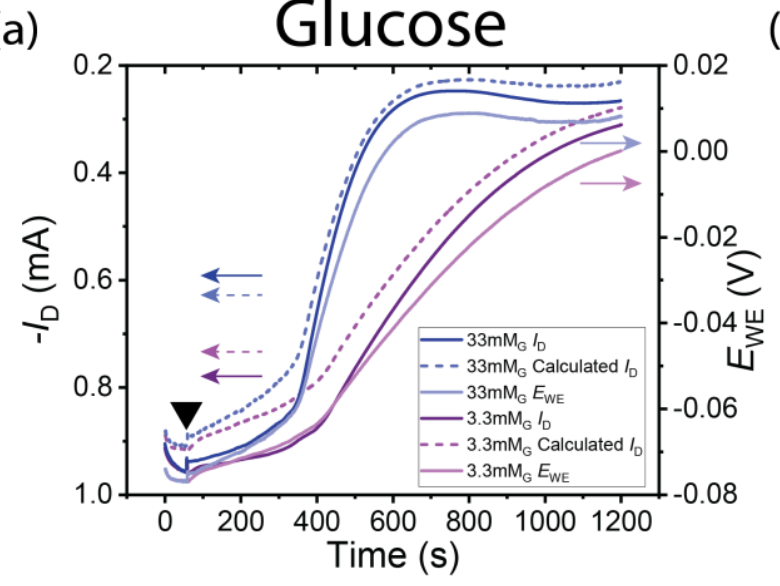

(b)

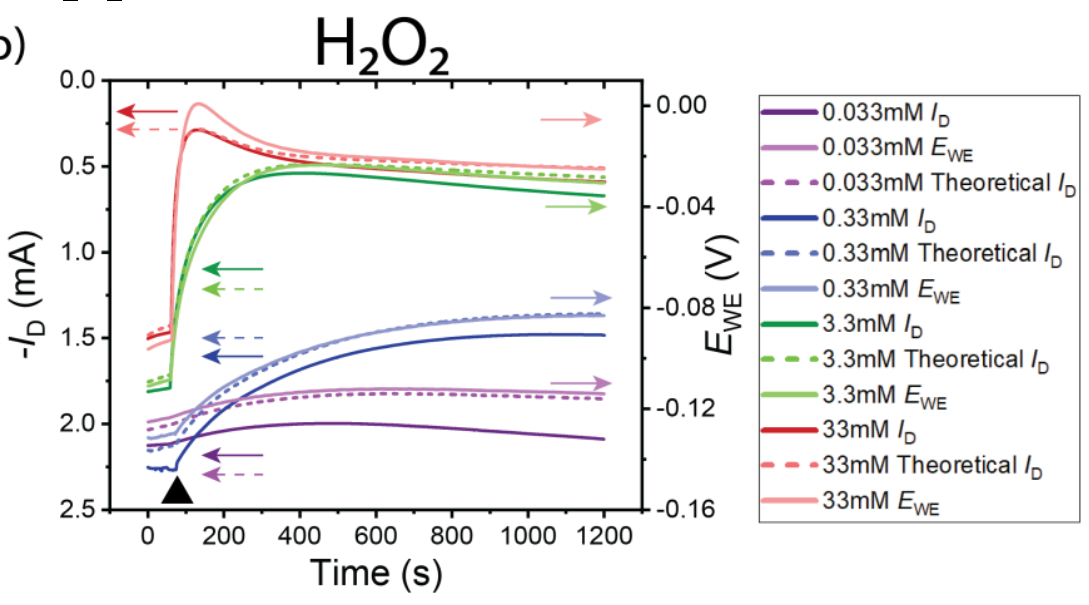

Figure S7. Performance of the p(gT2) FC - p(gT2) OECT device with $L=10 \mu \mathrm{m} ; W=2 \mathrm{~mm}$ and $\mathrm{d}=120 \mathrm{~nm}$. Dark solid lines: $I_{\mathrm{D}}$, light solid lines: $E_{\mathrm{WE}}$, dotted lines: calculated $I_{\mathrm{D}}$. Triangles indicate moment when fuel (glucose or $\mathrm{H}_{2} \mathrm{O}_{2}$ ) is added to $\mathrm{FC}$ (a) Addition of $3.3 \mathrm{mM}$ (purple) and $33 \mathrm{mM}$ (blue) glucose in PBS with GOx with $V_{\mathrm{D}}=-0.1 \mathrm{~V}$. (b) Increase in the concentration of $33 \mu \mathrm{M}$ (purple), $330 \mu \mathrm{M}$ (blue), $3.3 \mathrm{mM}$ (green)) and $\mathrm{H}_{2} \mathrm{O}_{2}\left(33 \mathrm{mM}\right.$ (red)) in PBS with $V_{\mathrm{D}}=-0.2 \mathrm{~V}$.

FC-OECT detection of lactate and ethanol

(a)

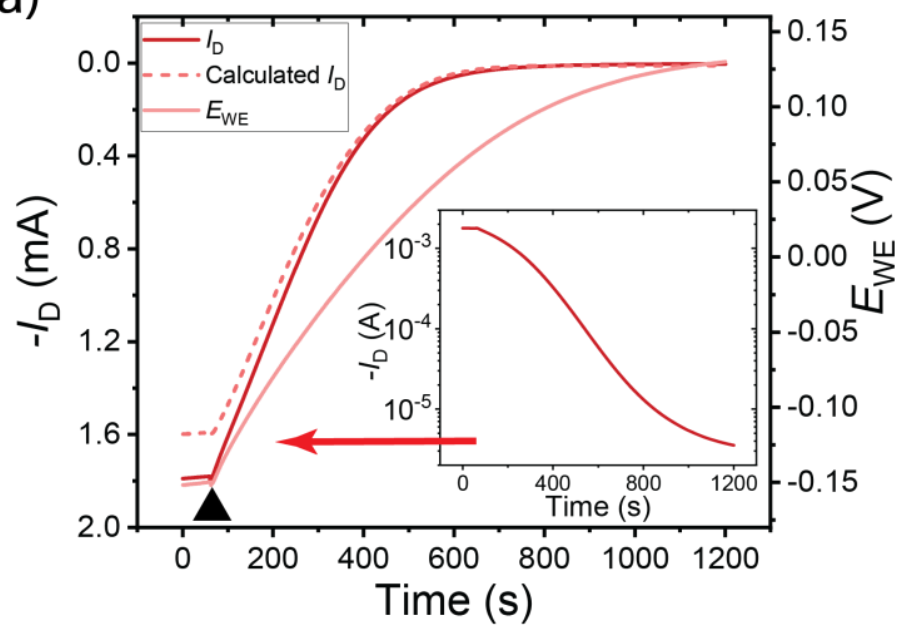

(b)

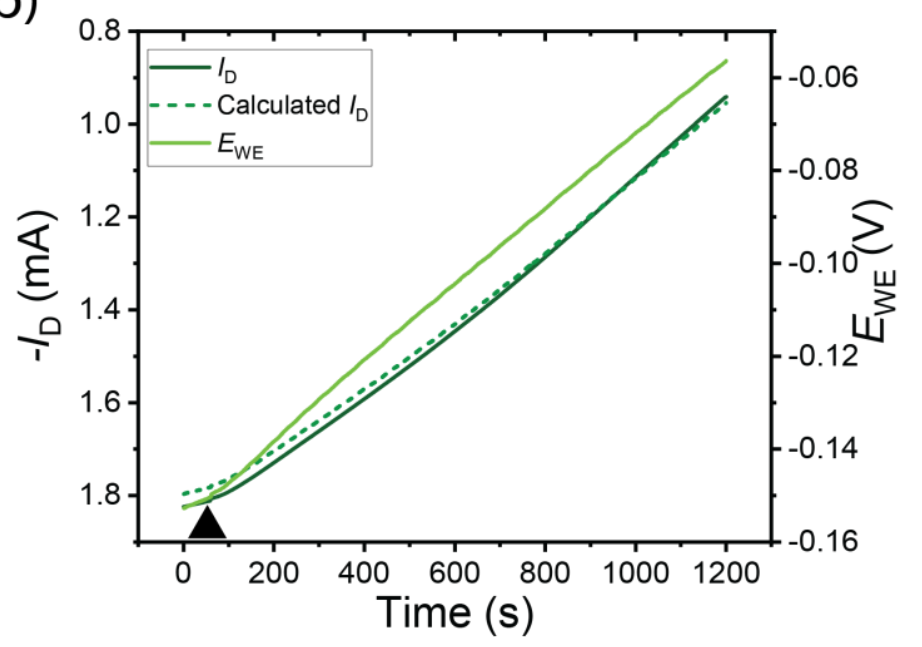

Figure S8. Detection of (a) lactate (33 mM lactate in lactate oxidase PBS solution) and (b) ethanol (33 mM ethanol in alcohol oxidase PBS solution) using $\mathrm{p}(\mathrm{gT} 2) \mathrm{FC}-\mathrm{p}(\mathrm{gT} 2)$ OECT device, $V_{\mathrm{D}}=-$ $0.2 \mathrm{~V}$. The inset shows the modulation of $I_{\mathrm{D}}$ on a log scale. 
$\underline{\text { Redox activity of } \mathrm{Ag} / \mathrm{AgCl} \text { pellet gate }}$

Large gate current peaks of up to $150 \mathrm{nA}$ are detected during recording of the transfer curve of $\mathrm{p}$ (gT2) (around $+0.1 \mathrm{~V}$ forward and $-0.1 \mathrm{~V}$ reversed scan) when a non-polarizable $\mathrm{Ag} / \mathrm{AgCl}$ pellet is used. This can be attributed to the redox-reaction of the $\mathrm{Ag} / \mathrm{AgCl}$ gate electrode $\left(\mathrm{Ag}\right.$ to $\mathrm{Ag}^{+}$ $(\mathrm{AgCl})$ and $\mathrm{Ag}^{+}$to $\left.\mathrm{Ag}\right)$. As a result of this, a constant current is measured (approximately $5 \mathrm{nA}$ ) during pulsing measurements to keep the OECT turned OFF (Fig. S9b). During this process, the channel material, p(gT2), performs ORR with molecular oxygen from ambient. When the OECT is gated using a purely capacitive gate material (Pt mesh), the defined redox-peaks are absent compared to $\mathrm{Ag} / \mathrm{AgCl}$. Furthermore, there is a large shift of the transfer curve to more positive potentials (lower turn $\mathrm{ON}$ voltages). Hence, larger positive potentials are required to turn the device OFF. Both OECTs were pulsed from $0 \mathrm{~V}$ to $+0.1 \mathrm{~V} V_{\mathrm{G}}$ to mimic the potential changes observed on the FC-OECT. As shown in Fig. S9d, the gate current during the pulsing experiment is also significantly lower compared to the $\mathrm{Ag} / \mathrm{AgCl}$ electrode. The difference of the two gate electrode materials is that while the $\mathrm{Ag} / \mathrm{AgCl}$ electrode can itself perform redox-reactions (similar to a metal electrode for energy storage devices), the Pt electrode likely drives electrochemical reactions with molecules of the electrolyte (e.g. ORR for $\mathrm{O}_{2}$ or oxygen evolution reactions (OER) or hydrogen evolution reaction (HER)), thus shifting the operational potentials to more positive potentials. To operate the FC-OECT efficiently, it is therefore required to use an OECT gate electrode that undergoes electrochemical redox-reactions at low potentials.

(a)

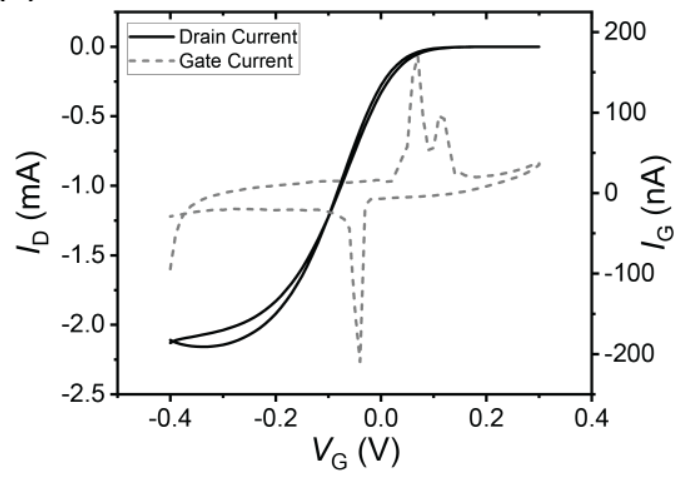

(c)

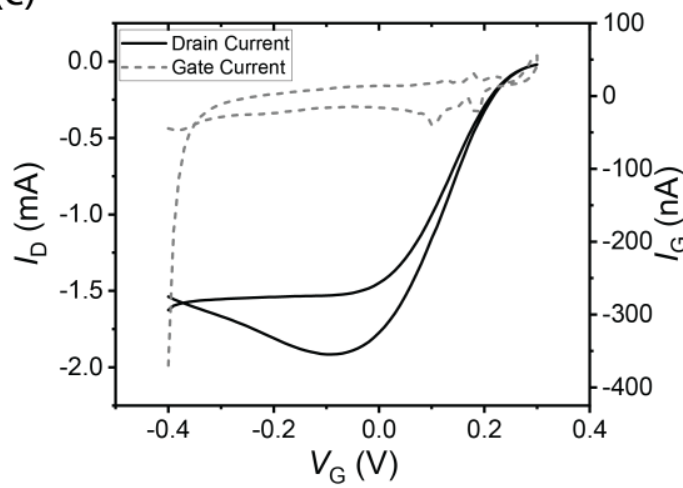

(b)

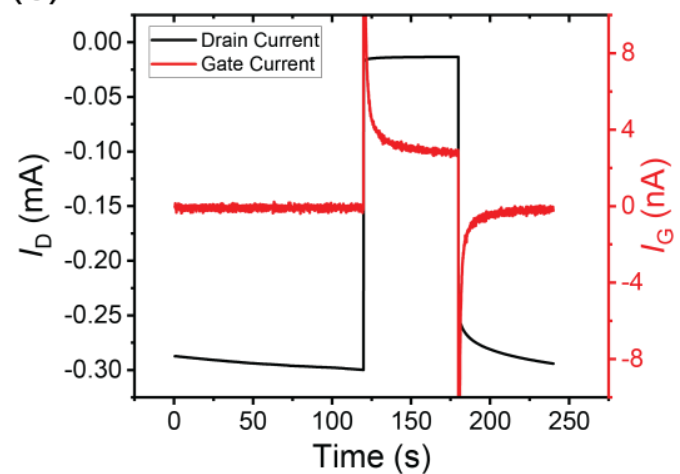

(d)

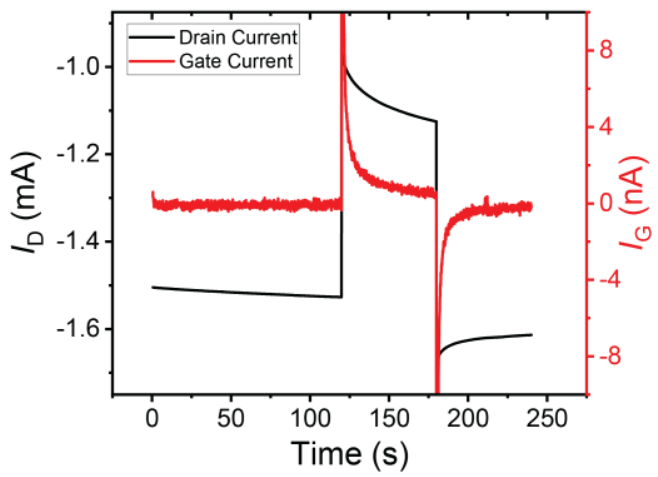

Figure S9. OECT characteristics of $\mathrm{p}(\mathrm{gT} 2)$ using PBS as the electrolyte. (a) Transfer curve $\left(\mathrm{V}_{\mathrm{D}}=-\right.$ $0.1 \mathrm{~V}$ ) with an $\mathrm{Ag} / \mathrm{AgCl}$ pellet as gate electrode (b) Pulsing experiment of an OECT with p(gT2) at $\mathrm{V}_{\mathrm{D}}=-0.1 \mathrm{~V}, V_{\mathrm{G}}=0 \mathrm{~V}$ to $+0.1 \mathrm{~V}$ with an $\mathrm{Ag} / \mathrm{AgCl}$ pellet as the gate electrode. (c) Transfer curve $\left(\mathrm{V}_{\mathrm{D}}=-0.1 \mathrm{~V}\right)$ with a Pt mesh as gate electrode (b) Pulsing of $\mathrm{p}(\mathrm{gT} 2)$ OECT at $\mathrm{V}_{\mathrm{D}}=-0.1 \mathrm{~V}, V_{\mathrm{G}}=0$ $\mathrm{V}$ to $+0.1 \mathrm{~V}$ with $\mathrm{Pt}$ mesh as the gate electrode. 
Thickness dependence of FC anode material

(a)

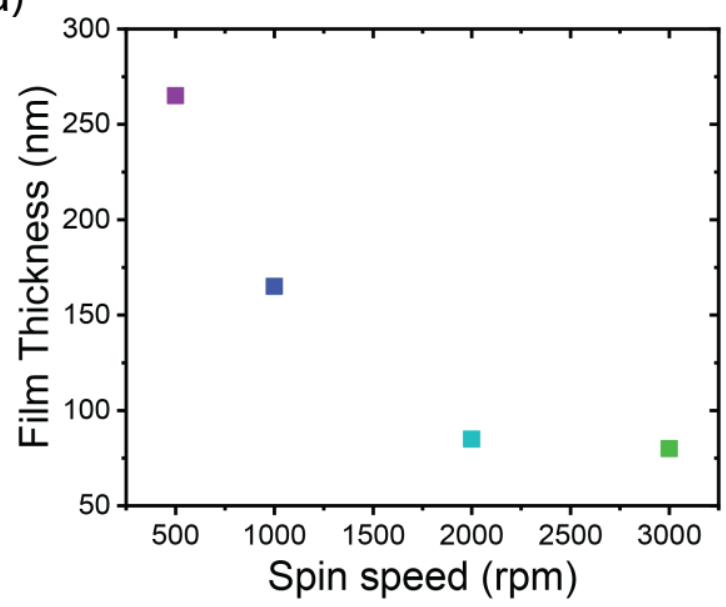

(c)

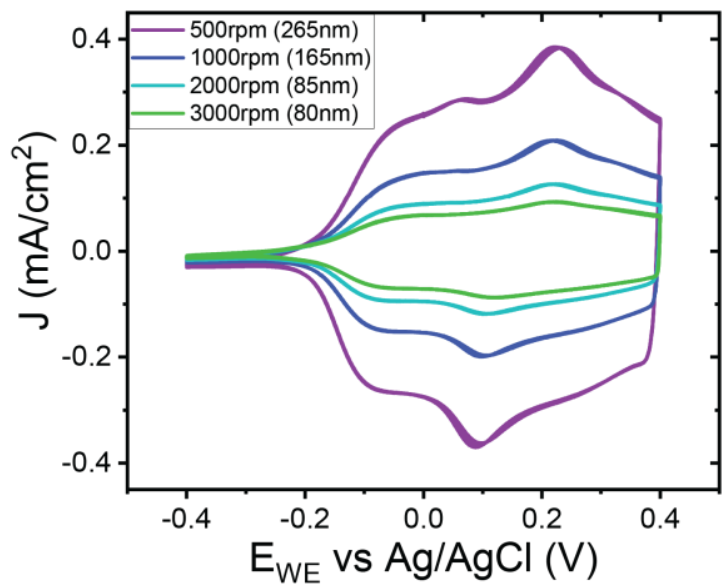

(b)

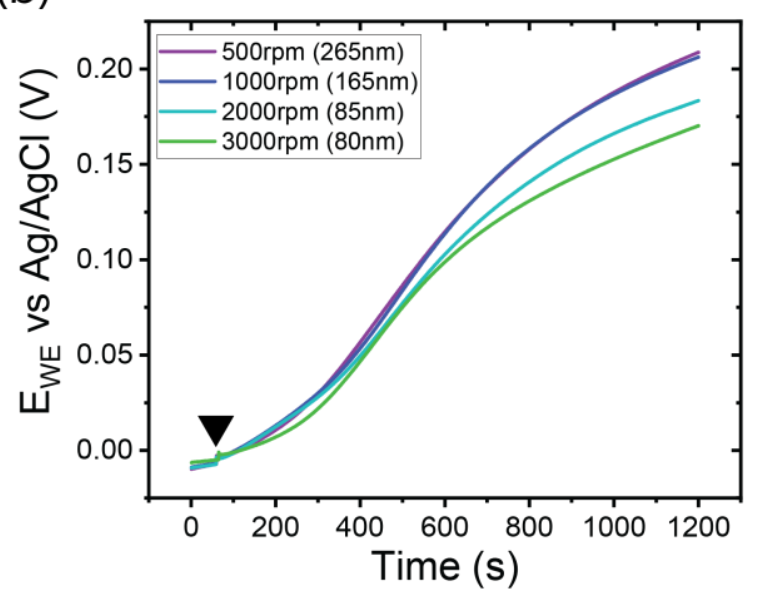

(d)

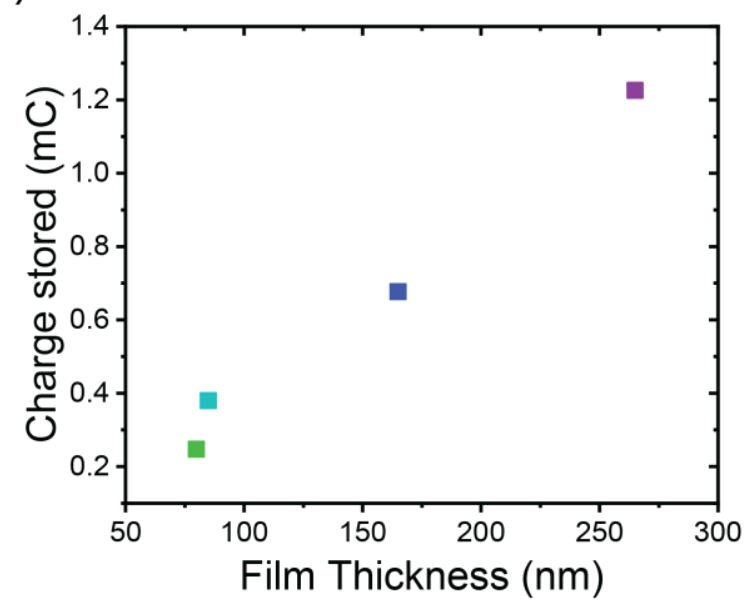

Figure S10. Thickness dependent charging measurements of p(gT2) on ITO coated glass substrates in PBS with glucose and GOx (via the intermediate $\mathrm{H}_{2} \mathrm{O}_{2}$ ). Thin films were prepared by spin coating polymer solution in chloroform $(10 \mathrm{mg} / \mathrm{mL})$ at different spin speeds. (a) Film thickness measurements for given spin speed. (b) OCV measurements of various sample thickness $(80 \mathrm{~nm}$ to $285 \mathrm{~nm}$ ). (c) Cyclic voltammetry with a scan rate of $50 \mathrm{mV} / \mathrm{s}$ and (d) accumulated charge from CA measurements by applying a constant potential to keep the polymer in its discharged (initial OCV) state for various thicknesses in PBS. 


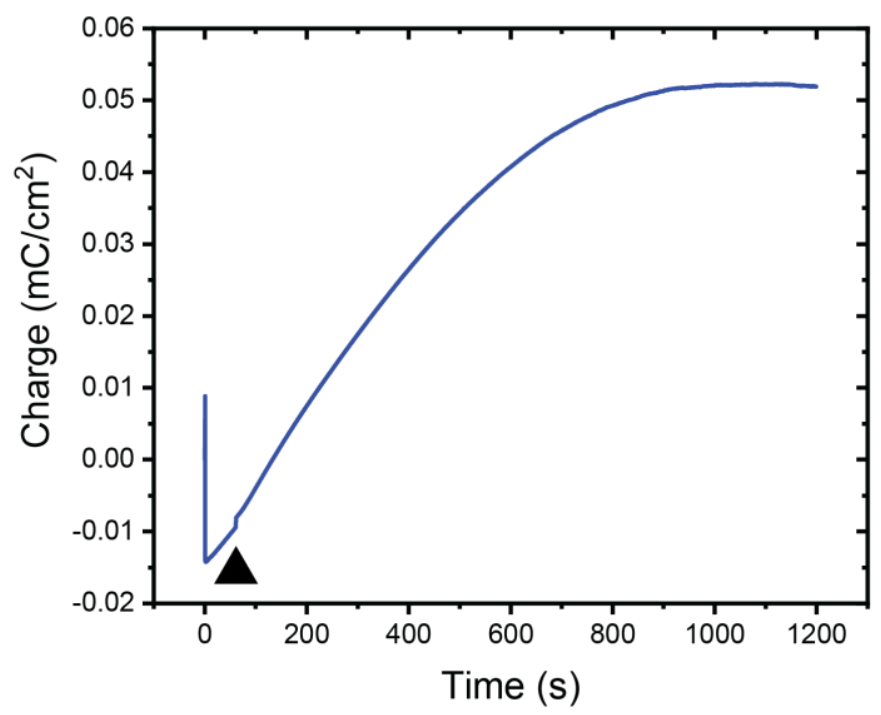

Figure S11. CA measurement of PEDOT:PSS on ITO-coated glass substrates with GOx and 33 $\mathrm{mM}$ Glucose for 20 minutes.

TTF-TCNQ as FC cathode

(a)<smiles>N#CC(C#N)=c1ccc(=C(C#N)C#N)cc1</smiles>

(d)

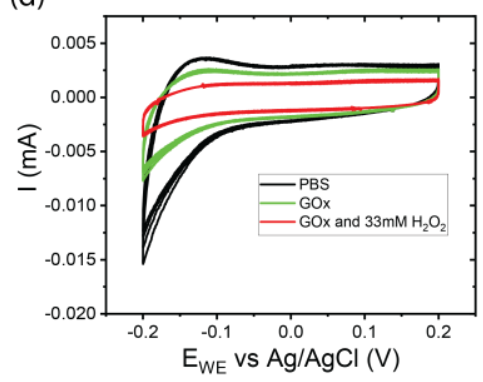

(b)

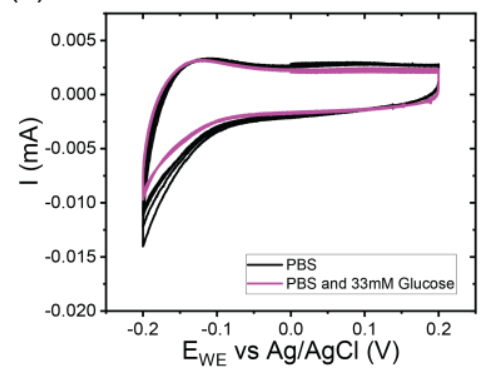

(e)

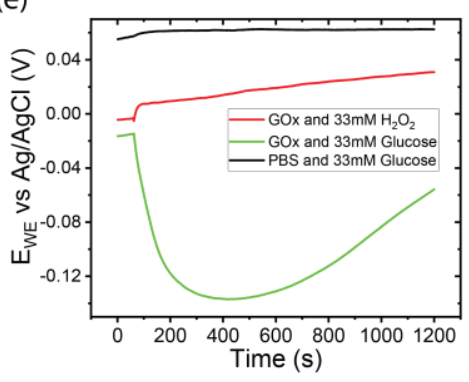

(c)

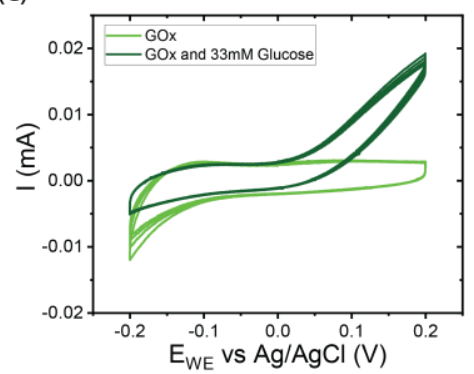

(f)

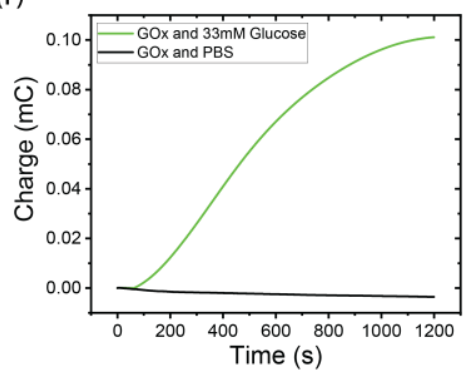

Figure S12. Electrochemical characterization of electrodes containing TTF-TCNQ. (a) Chemical structure of TTF-TCNQ. (b) CV measurements of electrodes made of TTF-TCNQ in PBS (black) and PBS and glucose (magenta), (c) after addition of $33 \mathrm{mM}$ glucose (dark green) to GOx (green) and (d) in GOx with $33 \mathrm{mM} \mathrm{H} \mathrm{O}_{2}$ (red). (e) OCV measurments of TTF-TCNQ electrode in GOx and glucose (green), GOx and $\mathrm{H}_{2} \mathrm{O}_{2}$ (red), PBS and glucose (black) for 20 minutes. (f) CA measurements of TTF-TCNQ with GOx and glucose (green), applying a contant potential of $0 \mathrm{~V}$ vs sat. $\mathrm{Ag} / \mathrm{AgCl}$ (initial OCV in PBS). Control measurement in absence of glucose in black. Note: $\mathrm{CV}$ measurement in (b) and CA measurements (f) indicate an electron transfer reation from GOx to TTF-TCNQ by the observation of a redox peak $>0 \mathrm{~V}$ vs $\mathrm{Ag} / \mathrm{AgCl}$ and measured charge injection from the electrolyte to the electrode. 
Evaluation of n-type polymer as candidate for FC cathode material

A recent publication (28) claimed the observation of spontaneous electron transfer from the reduced GOx enzyme to conjugated polymers with large EA (n-type polymer, e.g. p(gNDI-T2)-90 (P-90) (39)). These results are in contradiction to our findings. Measurements presented in Fig. S13 show that there is no spontaneous, direct ET from the reduced enzyme to of the polymer $\mathrm{p}(\mathrm{gNDI}-\mathrm{T} 2)$ 90 when deposited on ITO coated electrodes. Similar findings were also observed for other reports for n-type polymers, where charged (reduced) n-type polymers rapidly undergo ORR with molecular oxygen in ambient conditions (1).

As shown in Fig. S13a, electrodes coated with the polymer p(gNDI-T2)-90 (P-90) (39) show that no spontaneous charging of the $n$-type polymer is observed after the addition of glucose/GOx (Fig. $\mathrm{S} 13 \mathrm{a})$ and the response is similar to the one observed for the addition of $\mathrm{H}_{2} \mathrm{O}_{2}$ to a PBS solution. The cyclic voltammogram is shown in Fig. S13b and other publications $(39,48)$, showing that the polymer lacks oxidation states below $+0.4 \mathrm{~V}$ vs $\mathrm{Ag} / \mathrm{AgCl}$, meaning that the polymer does not become charged during the enzymatic reaction showing in Fig. 13a. During the electrochemical reduction (Fig. 13b), one can observe that electrons injected in the n-type polymer are more likely to be lost when oxidation agents are present such as $\mathrm{H}_{2} \mathrm{O}_{2}$ (visible by a drop of the magnitude of the oxidation peak during the reduction/oxidation of the $n$-type polymer)

(a)

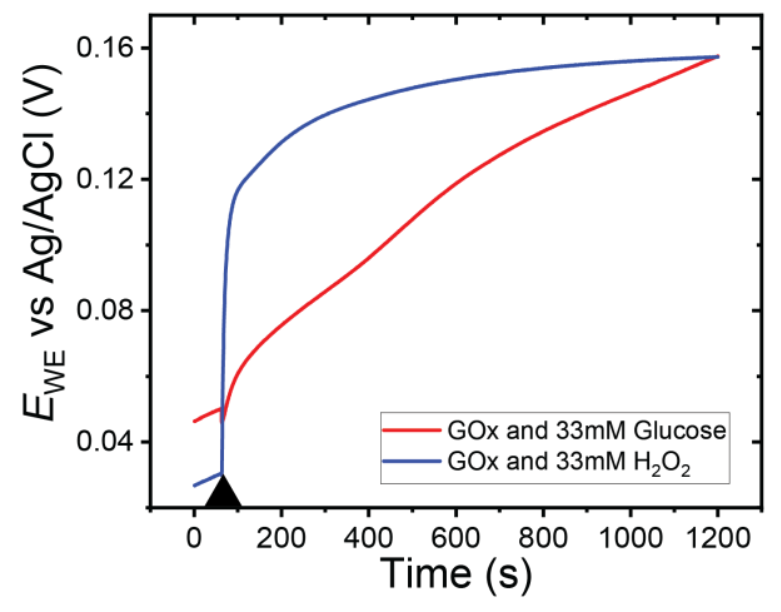

(b)

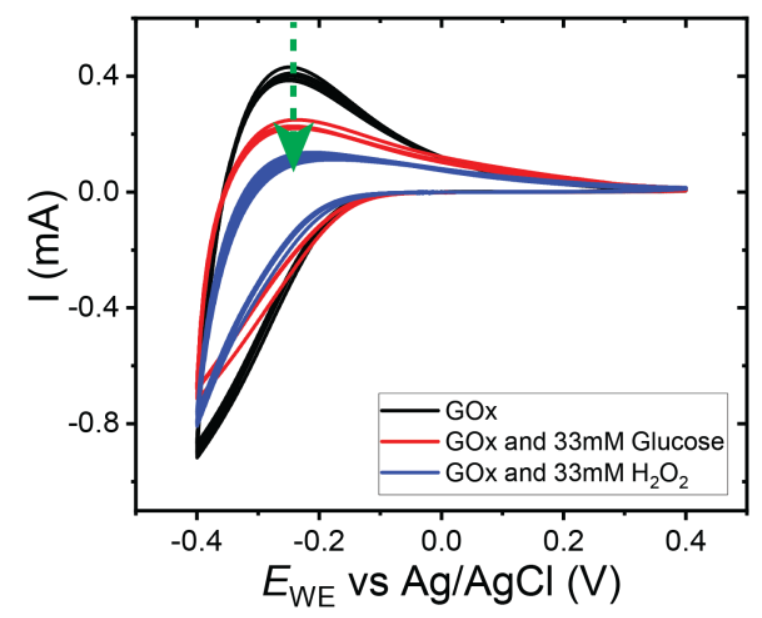

Figure S13. Electrochemical characterization of the n-type polymer p(NDI-T2)-90 (P-90) on ITO coated glass substrates in PBS (a) Monitoring of the OCV with $33 \mathrm{mM}$ glucose/GOx (red) or 33 $\mathrm{mM} \mathrm{H} \mathrm{O}_{2}$ (blue). Triangle indicate the addition of glucose or $\mathrm{H}_{2} \mathrm{O}_{2}$ (b) $\mathrm{CV}$ measurements with $\mathrm{GOx}$ (black), GOx and $33 \mathrm{mM}$ glucose (red) and $\mathrm{GOx}$ and $33 \mathrm{mM} \mathrm{H} \mathrm{O}_{2}$. Green arrow indicates drop in amount of electrons extracted from the reverse CV scan. 

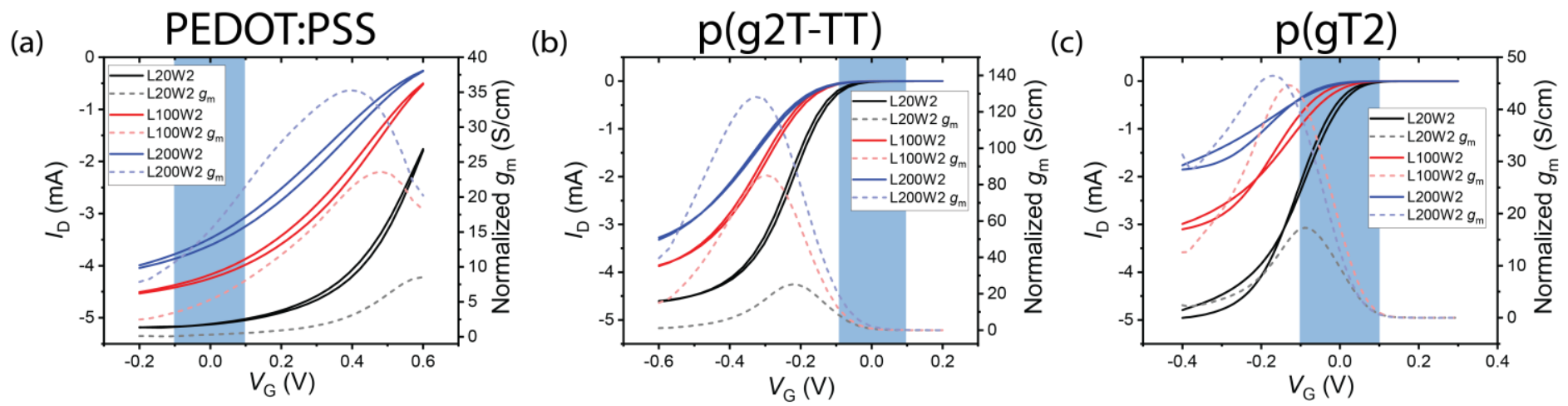

Figure S14. Characteristics of OECTs with PEDOT:PSS, p(g2T-TT) and $\mathrm{p}(\mathrm{gT} 2)$ as the channel materials. The channel length was varied (fixed width $(W)$ of $2 \mathrm{~mm}$, corresponding to $\mathrm{W} 2$ ) to modify the channel dimensions as the following: $L=20,100$ or $200 \mu \mathrm{m}$ corresponding to L20W2, L100W2 and L200W2. Transfer curves of the OECTs are measured with $V_{\mathrm{D}}=-0.2 \mathrm{~V}$ and a scan rate of $0.6 \mathrm{~V} / \mathrm{s}$ (solid lines) and the normalized transconductance $\left(g_{m}, n_{\text {norm }}=W \mathrm{~d} / L\right)$ is shown as dashed lines for (a) PEDOT:PSS, (b) p(g2T-TT) and (c) p(gT2) respectively. Shaded regions correspond to range of potential change of fuel cell. 
Table S1. Fuel Cell-OECT performance with p(gT2) OECT aspect ratio $W / L=200$ : Effect of Fuel Cell anode material

\begin{tabular}{|c|c|c|c|c|c|c|}
\hline Experiment & $\begin{array}{c}\text { Fuel Cell } \\
\text { Anode }\end{array}$ & $\begin{array}{c}\text { OECT } \\
\text { Channel }\end{array}$ & $V_{\mathrm{D}}(\mathrm{V})$ & $\begin{array}{l}\text { Analyte } \\
(33 \mathrm{mM})\end{array}$ & $\begin{array}{l}\text { Maximum } \\
\Delta E_{\mathrm{WE}}(\mathrm{V})\end{array}$ & $\begin{array}{l}\text { Maximum } \\
\Delta I_{\mathrm{D}}(\mathrm{mA})\end{array}$ \\
\hline 1 & $\mathrm{p}(\mathrm{gT} 2)$ & $\overline{p(g T 2)}$ & -0.1 & Glucose & 0.064 & 1.39 \\
\hline 2 & $p(g T 2)$ & $p(g T 2)$ & -0.1 & $\mathrm{H}_{2} \mathrm{O}_{2}$ & 0.067 & 0.773 \\
\hline 3 & $p$ (gT2) & p(gT2) & -0.2 & Glucose & 0.077 & 0.861 \\
\hline 4 & $p($ gT2) & $\mathrm{p}(\mathrm{gT} 2)$ & -0.2 & $\mathrm{H}_{2} \mathrm{O}_{2}$ & 0.127 & 2.13 \\
\hline 5 & $\begin{array}{c}\mathrm{p}(\mathrm{gPyDPP}- \\
\text { MeOT2) }\end{array}$ & $\mathrm{p}(\mathrm{gT} 2)$ & -0.1 & Glucose & 0.059 & 0.174 \\
\hline 6 & $\begin{array}{c}\text { p(gPyDPP- } \\
\text { MeOT2) }\end{array}$ & $\mathrm{p}(\mathrm{gT} 2)$ & -0.1 & $\mathrm{H}_{2} \mathrm{O}_{2}$ & 0.026 & 0.212 \\
\hline 7 & $\begin{array}{c}\text { p(gPyDPP- } \\
\text { MeOT2) }\end{array}$ & $\mathrm{p}(\mathrm{gT} 2)$ & -0.2 & Glucose & 0.0489 & 0.450 \\
\hline 8 & $\begin{array}{c}\text { p(gPyDPP- } \\
\text { MeOT2) }\end{array}$ & $\mathrm{p}(\mathrm{gT} 2)$ & -0.2 & $\mathrm{H}_{2} \mathrm{O}_{2}$ & 0.0589 & 0.761 \\
\hline
\end{tabular}

The fuel cell anode with larger reaction current $(\mathrm{p}(\mathrm{gT} 2))$ leads to greater current modulation on the OECT.

Table S2. Fuel Cell-OECT performance: Effect of OECT Channel Material; $W / L=100$

\begin{tabular}{|c|c|c|c|c|c|c|}
\hline Experiment & $\begin{array}{c}\text { Fuel Cell } \\
\text { Anode }\end{array}$ & $\begin{array}{c}\text { OECT } \\
\text { Channel }\end{array}$ & $V_{\mathrm{D}}(\mathrm{V})$ & $\begin{array}{l}\text { Analyte } \\
(33 \mathrm{mM})\end{array}$ & $\begin{array}{l}\text { Maximum } \\
\Delta E_{\mathrm{wE}}(\mathrm{V})\end{array}$ & $\begin{array}{l}\text { Maximum } \\
\Delta I_{\mathrm{D}}(\mathrm{mA})\end{array}$ \\
\hline 9 & $\mathrm{p}(\mathrm{gT} 2)$ & $\mathrm{p}(\mathrm{gT} 2)$ & -0.1 & Glucose & 0.0406 & 0.543 \\
\hline 10 & $\mathrm{p}(\mathrm{gT} 2)$ & $\mathrm{p}(\mathrm{gT} 2)$ & -0.1 & $\mathrm{H}_{2} \mathrm{O}_{2}$ & 0.0615 & 0.611 \\
\hline 11 & $\mathrm{p}(\mathrm{gT} 2)$ & $\mathrm{p}(\mathrm{gT} 2)$ & -0.2 & Glucose & 0.0339 & 0.620 \\
\hline 12 & $\mathrm{p}(\mathrm{gT} 2)$ & $\mathrm{p}(\mathrm{gT} 2)$ & -0.2 & $\mathrm{H}_{2} \mathrm{O}_{2}$ & 0.0524 & 1.13 \\
\hline 13 & $\mathrm{p}(\mathrm{gT} 2)$ & $\mathrm{p}(\mathrm{g} 2 \mathrm{~T}-\mathrm{TT})$ & -0.1 & Glucose & 0.0371 & 0.008 \\
\hline 14 & $\mathrm{p}(\mathrm{gT} 2)$ & $\mathrm{p}(\mathrm{g} 2 \mathrm{~T}-\mathrm{TT})$ & -0.1 & $\mathrm{H}_{2} \mathrm{O}_{2}$ & 0.065 & 0.021 \\
\hline 15 & $\mathrm{p}(\mathrm{gT} 2)$ & $\mathrm{p}(\mathrm{g} 2 \mathrm{~T}-\mathrm{TT})$ & -0.2 & Glucose & 0.102 & 0.408 \\
\hline 16 & $\mathrm{p}(\mathrm{gT} 2)$ & $\mathrm{p}(\mathrm{g} 2 \mathrm{~T}-\mathrm{TT})$ & -0.2 & $\mathrm{H}_{2} \mathrm{O}_{2}$ & 0.13 & 0.845 \\
\hline 17 & $p(g T 2)$ & PEDOT:PSS & -0.1 & Glucose & 0.139 & 0.04 \\
\hline 18 & $\mathrm{p}(\mathrm{gT} 2)$ & PEDOT:PSS & -0.1 & $\mathrm{H}_{2} \mathrm{O}_{2}$ & 0.1 & 0.02 \\
\hline 19 & $\mathrm{p}$ (gT2) & PEDOT:PSS & -0.2 & Glucose & 0.184 & 0.031 \\
\hline 20 & $\mathrm{p}(\mathrm{gT} 2)$ & PEDOT:PSS & -0.2 & $\mathrm{H}_{2} \mathrm{O}_{2}$ & 0.113 & 0.0849 \\
\hline
\end{tabular}

$\mathrm{p}$ (gT2) OECT gives the best overall performance as the oxidation window of the fuel cell overlaps the best with the range of max transconductance of the OECT.

Table S3. Fuel Cell-OECT performance with p(gT2) on both fuel cell anode and OECT channel: Effect of OECT Channel Aspect Ratio 


\begin{tabular}{|c|c|c|c|c|c|}
\hline Experiment & $\begin{array}{c}\text { Channel } \\
\text { Aspect Ratio } \\
(\mathrm{W} / \mathrm{L})\end{array}$ & $V_{\mathrm{D}}(\mathrm{V})$ & $\begin{array}{c}\text { Analyte } \\
(33 \mathrm{mM})\end{array}$ & $\begin{array}{c}\text { Maximum } \\
\Delta E_{\mathrm{WE}}(\mathrm{V})\end{array}$ & $\begin{array}{c}\text { Maximum } \\
\Delta I_{\mathrm{D}}(\mathrm{mA})\end{array}$ \\
\hline 1 & 200 & -0.1 & Glucose & 0.064 & 1.39 \\
\hline 2 & 200 & -0.1 & $\mathrm{H}_{2} \mathrm{O}_{2}$ & 0.067 & 0.773 \\
\hline 3 & 200 & $-\mathbf{0 . 2}$ & $\mathrm{Glucose}$ & 0.077 & 0.861 \\
\hline 4 & 200 & $-\mathbf{0 . 2}$ & $\mathrm{H}_{2} \mathrm{O}_{2}$ & 0.127 & 2.13 \\
\hline 9 & 100 & -0.1 & $\mathrm{Glucose}$ & 0.0406 & 0.543 \\
\hline 10 & 100 & -0.1 & $\mathrm{H}_{2} \mathrm{O}_{2}$ & 0.0274 & 0.174 \\
\hline 11 & 100 & $\mathbf{- 0 . 2}$ & $\mathrm{Glucose}_{2}$ & 0.0339 & 0.620 \\
\hline 12 & 100 & $\mathbf{- 0 . 2}$ & $\mathrm{H}_{2} \mathrm{O}_{2}$ & 0.0524 & 1.13 \\
\hline
\end{tabular}

Using a larger $W / L$ ratio of the $\mathrm{p}(\mathrm{gT} 2)$ OECT leads to greater current modulation. 
Note: Figures S15-S19 correspond to experiments summarized in Tables 1 to 3.

(a)

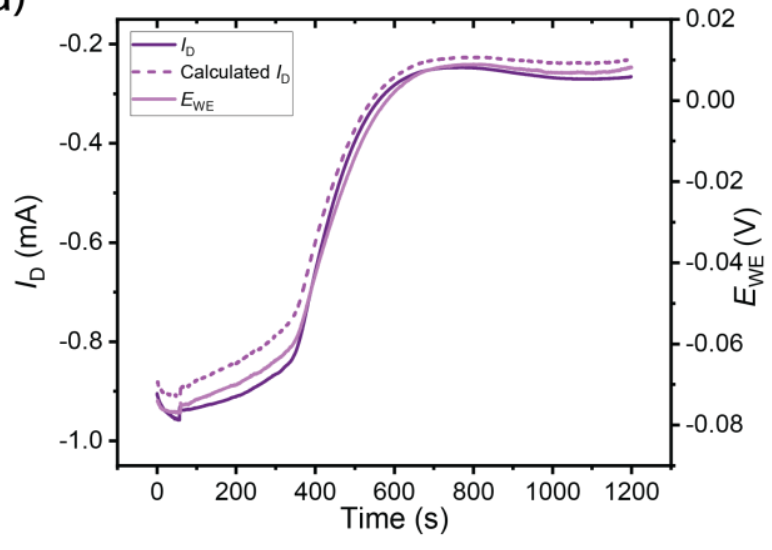

(c)

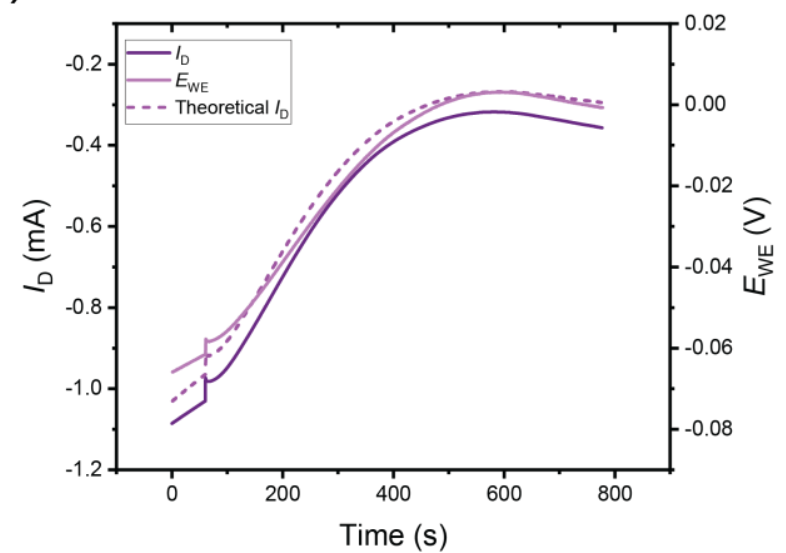

(b)

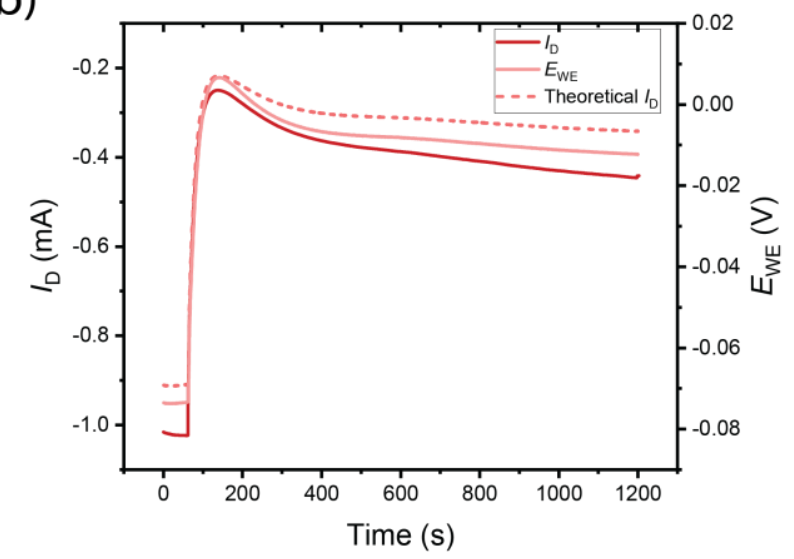

(d)

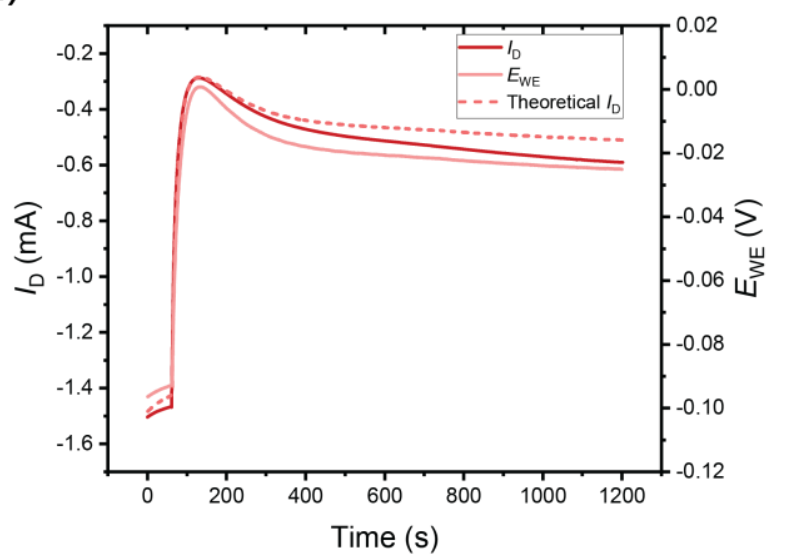

Figure S15. p(gT2) FC - p(gT2) OECT L10W2. (a) $33 \mathrm{mM}$ glucose and GOx, $\mathrm{V}_{\mathrm{D}}=-0.1 \mathrm{~V}$ (b) 33 $\mathrm{mM} \mathrm{H} \mathrm{H}_{2} \mathrm{O}_{2}$ and PBS, $\mathrm{V}_{\mathrm{D}}=-0.1 \mathrm{~V}$ (c) $33 \mathrm{mM}$ glucose and $\mathrm{GOx}, \mathrm{V}_{\mathrm{D}}=-0.2 \mathrm{~V}$ (d) $33 \mathrm{mM} \mathrm{H}_{2} \mathrm{O}_{2}$ and PBS, $\mathrm{V}_{\mathrm{D}}=-0.2 \mathrm{~V}$ 
(a)

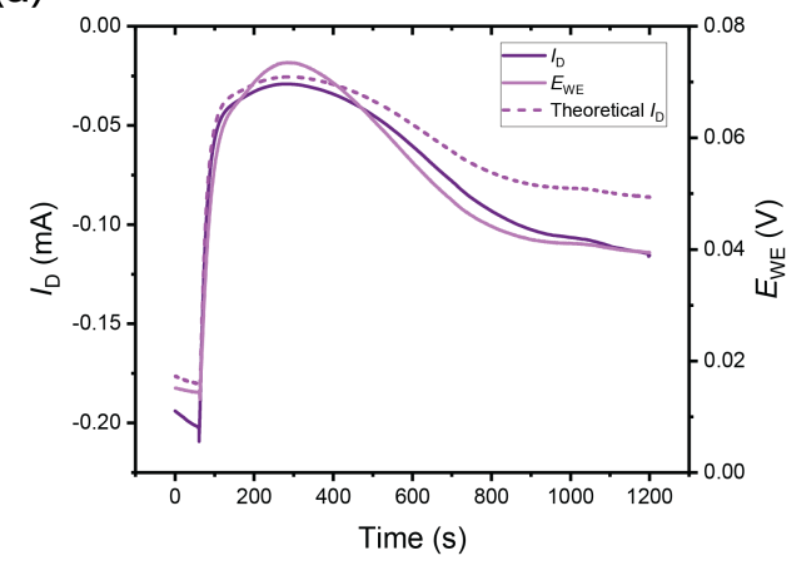

(b)

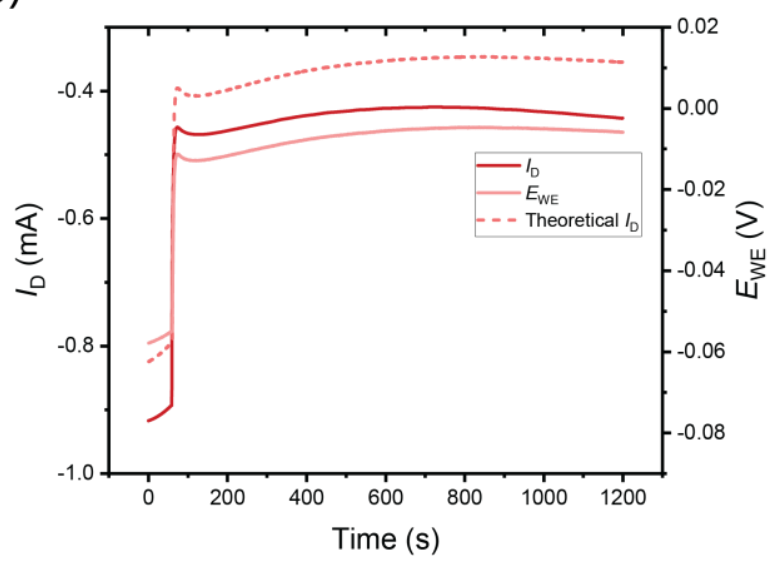

(d)

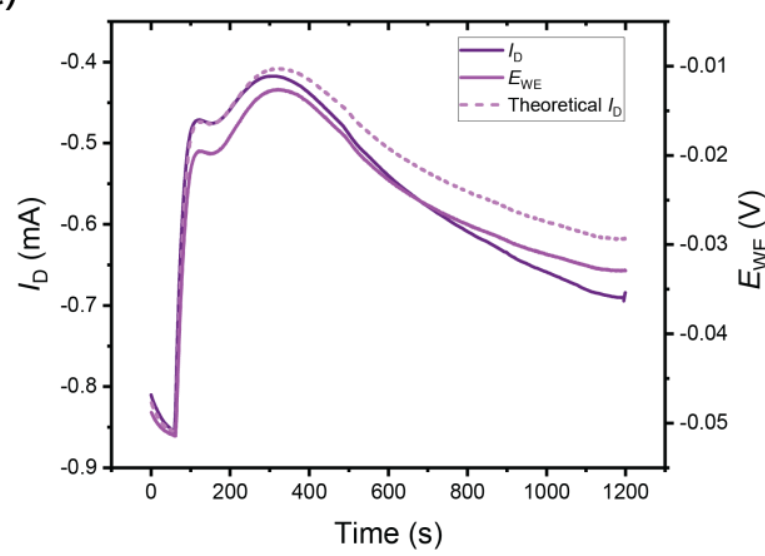

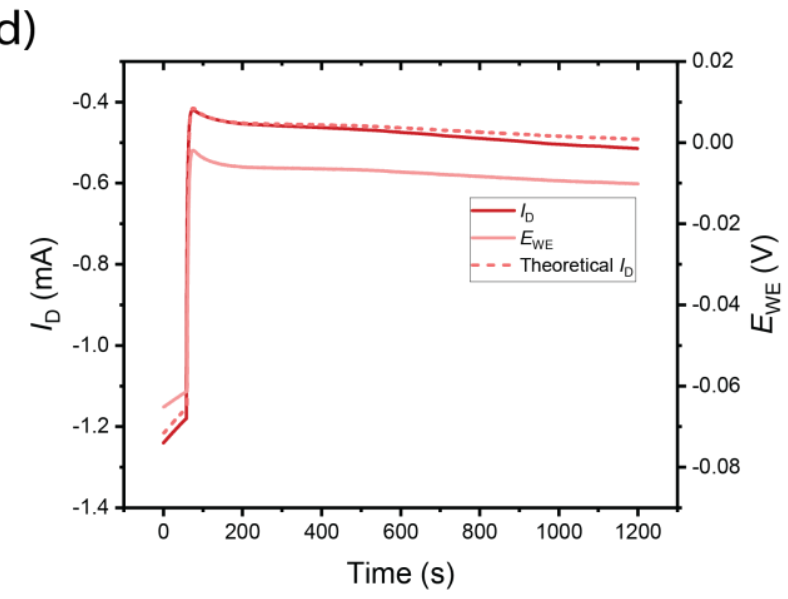

Figure S16. $\mathrm{p}$ (gPyDPP-MeOT2) FC $-\mathrm{p}(\mathrm{gT2})$ OECT L10W2. (a) $33 \mathrm{mM}$ glucose and GOx, $\mathrm{D}=-$ $0.1 \mathrm{~V}$ (b) $33 \mathrm{mM} \mathrm{H} \mathrm{O}_{2}$ and $\mathrm{PBS}, \mathrm{V}_{\mathrm{D}}=-0.1 \mathrm{~V}$ (c) $33 \mathrm{mM}$ glucose and $\mathrm{GOx}, \mathrm{V}_{\mathrm{D}}=-0.2 \mathrm{~V}$ (d) $33 \mathrm{mM}$ $\mathrm{H}_{2} \mathrm{O}_{2}$ and $\mathrm{PBS}, \mathrm{V}_{\mathrm{D}}=-0.2 \mathrm{~V}$ 
(a)

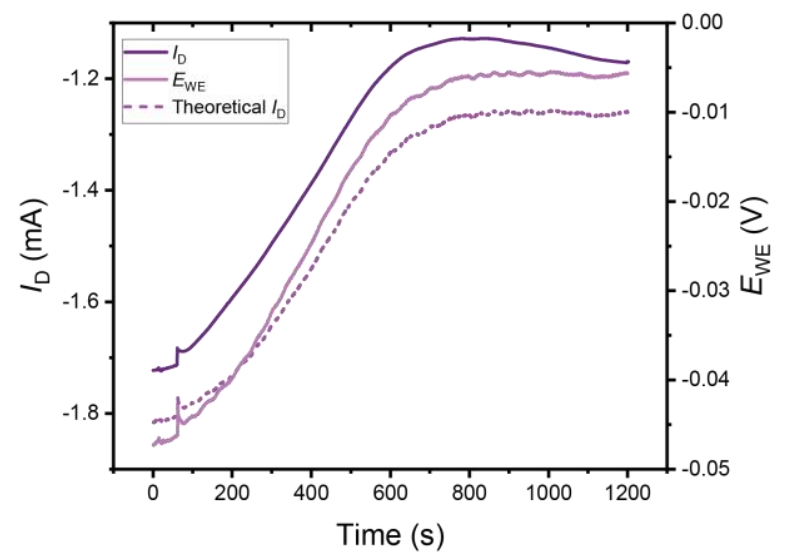

(c)

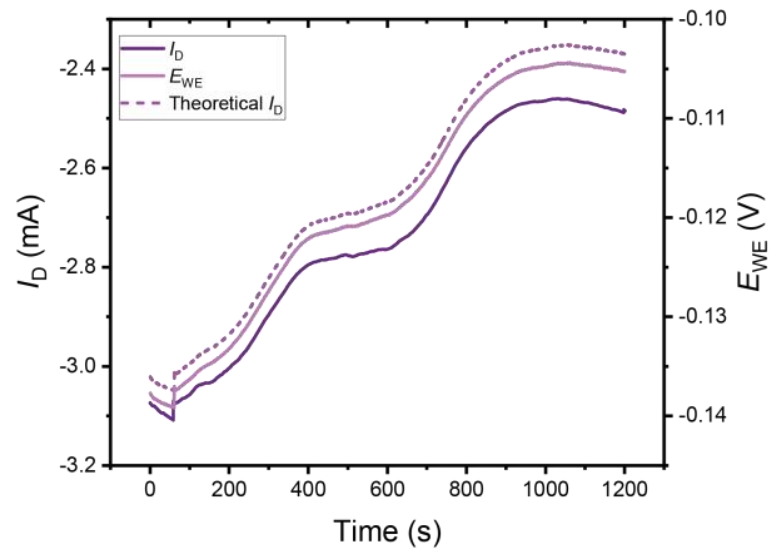

(b)

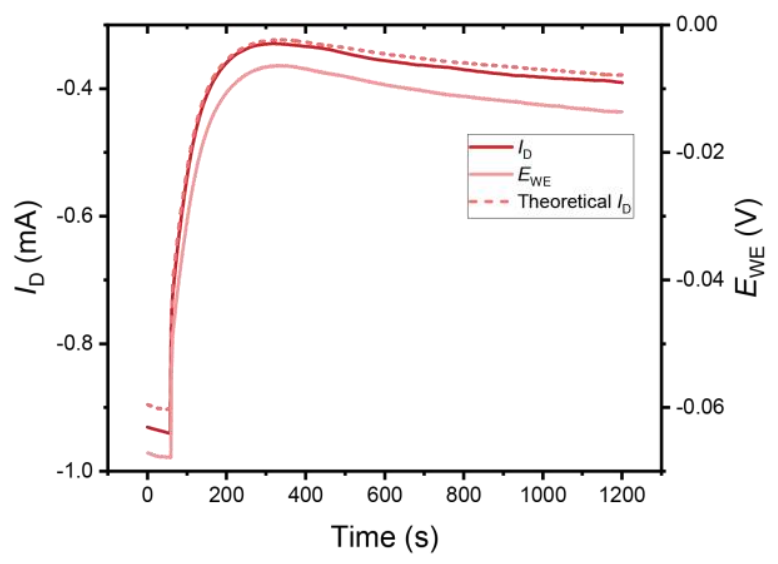

(d)

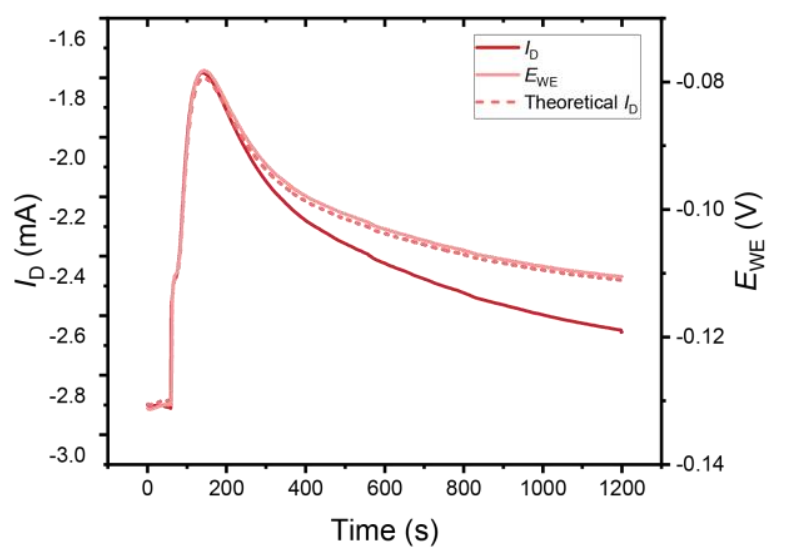

Figure S17. p(gT2) FC - p(gT2) OECT L20W2. (a) $33 \mathrm{mM}$ glucose and GOx, $\mathrm{V}_{\mathrm{D}}=-0.1 \mathrm{~V}$ (b) 33 $\mathrm{mM} \mathrm{H} \mathrm{H}_{2} \mathrm{O}_{2}$ and PBS, $\mathrm{V}_{\mathrm{D}}=-0.1 \mathrm{~V}$ (c) $33 \mathrm{mM}$ glucose and $\mathrm{GOx}, \mathrm{V}_{\mathrm{D}}=-0.2 \mathrm{~V}$ (d) $33 \mathrm{mM} \mathrm{H}_{2} \mathrm{O}_{2}$ and PBS, $\mathrm{V}_{\mathrm{D}}=-0.2 \mathrm{~V}$ 
(a)

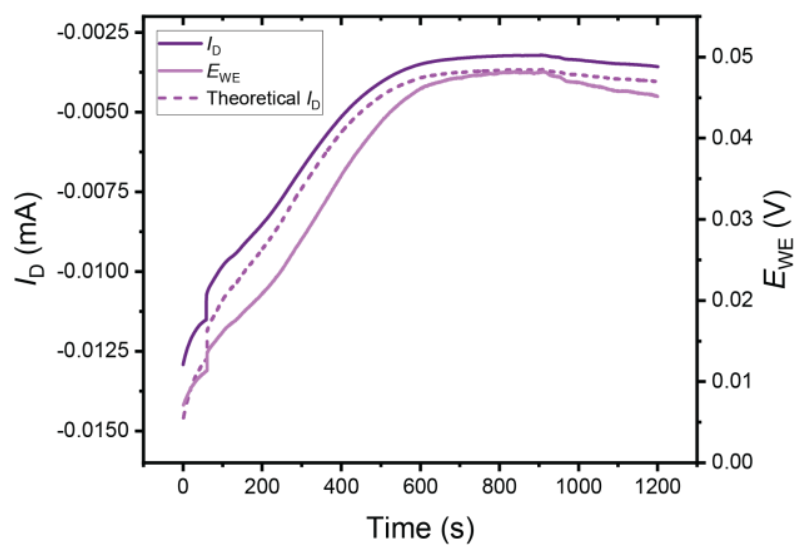

(c)

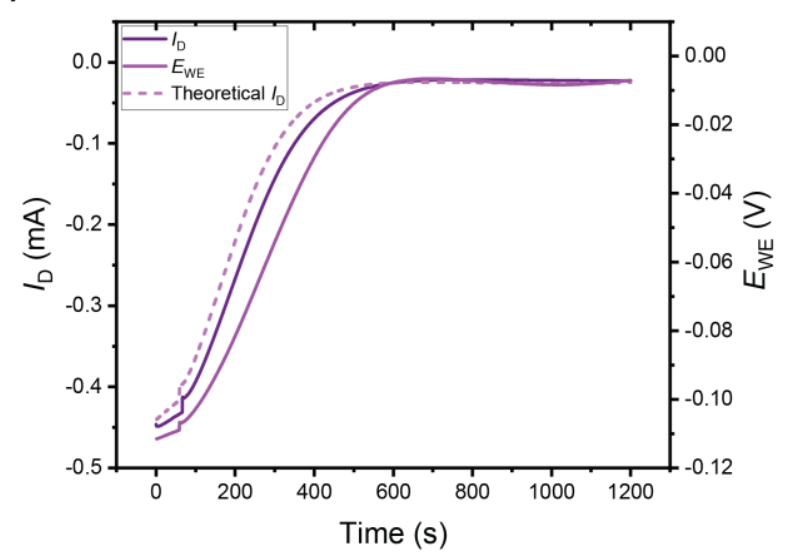

(b)

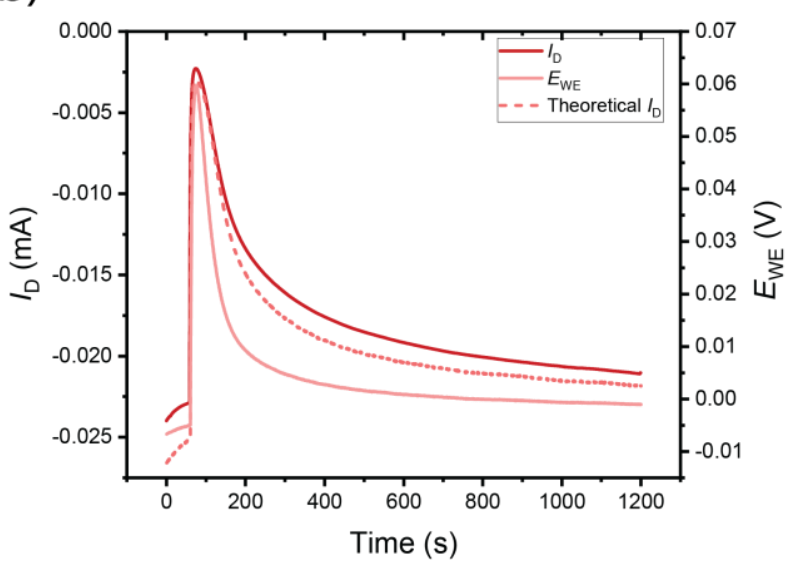

(d)

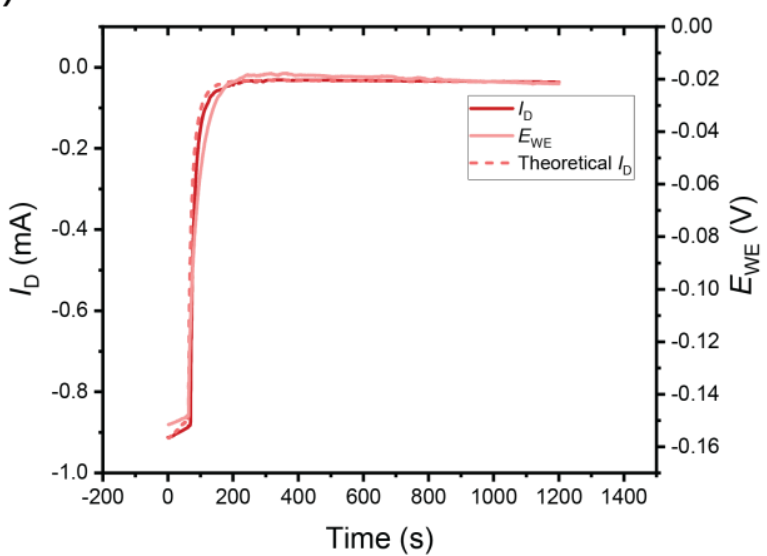

Figure S18. p(gT2) FC $-\mathrm{p}(\mathrm{g} 2 \mathrm{~T}-\mathrm{TT})$ OECT L20W2. (a) $33 \mathrm{mM}$ glucose and GOx, $\mathrm{V}_{\mathrm{D}}=-0.1 \mathrm{~V}$ (b) $33 \mathrm{mM} \mathrm{H}_{2} \mathrm{O}_{2}$ and PBS, $\mathrm{V}_{\mathrm{D}}=-0.1 \mathrm{~V}$ (c) $33 \mathrm{mM}$ glucose and $\mathrm{GOx}, \mathrm{V}_{\mathrm{D}}=-0.2 \mathrm{~V}$ (d) $33 \mathrm{mM} \mathrm{H}_{2} \mathrm{O}_{2}$ and PBS, $\mathrm{V}_{\mathrm{D}}=-0.2 \mathrm{~V}$ 
(a)

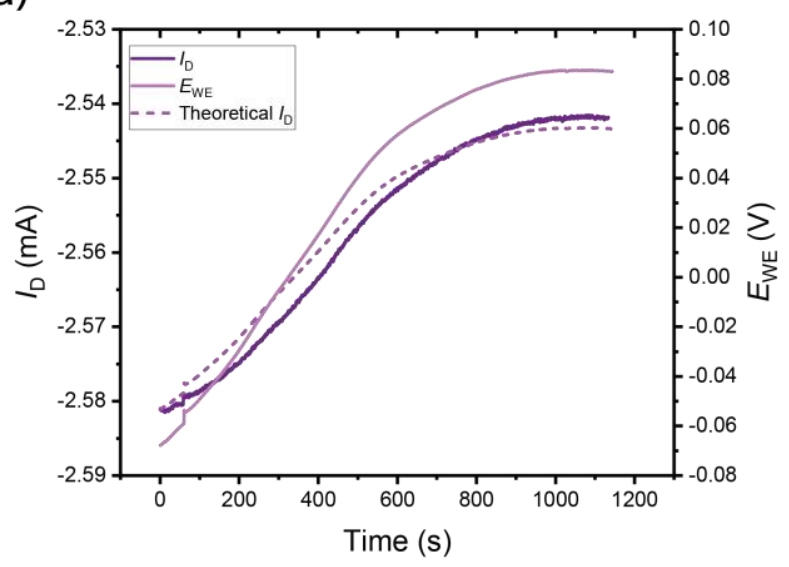

(c)

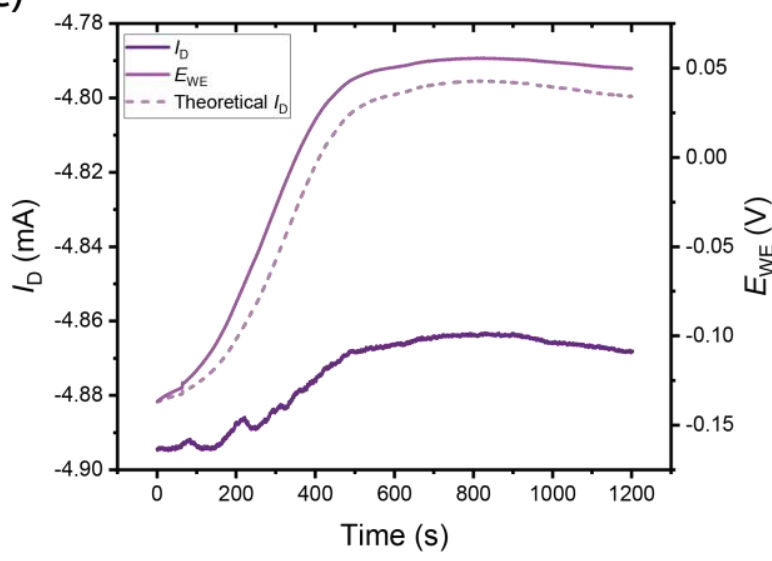

(b)

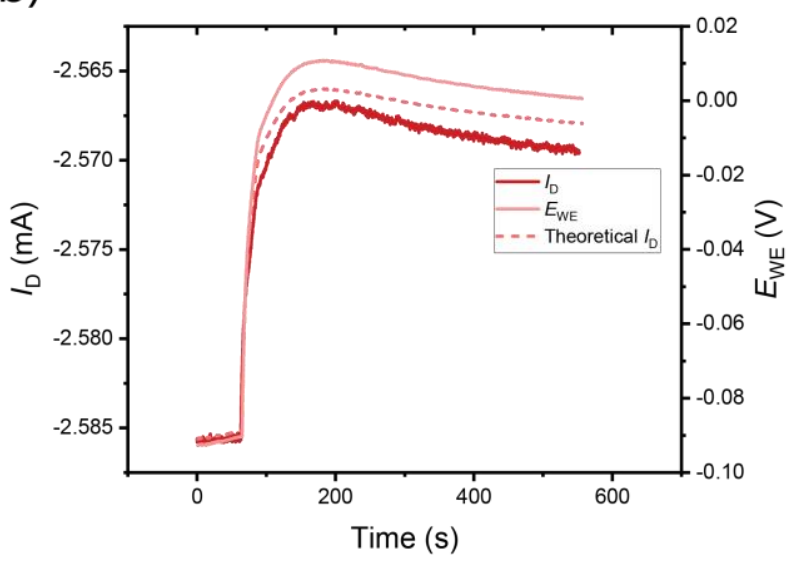

(d)

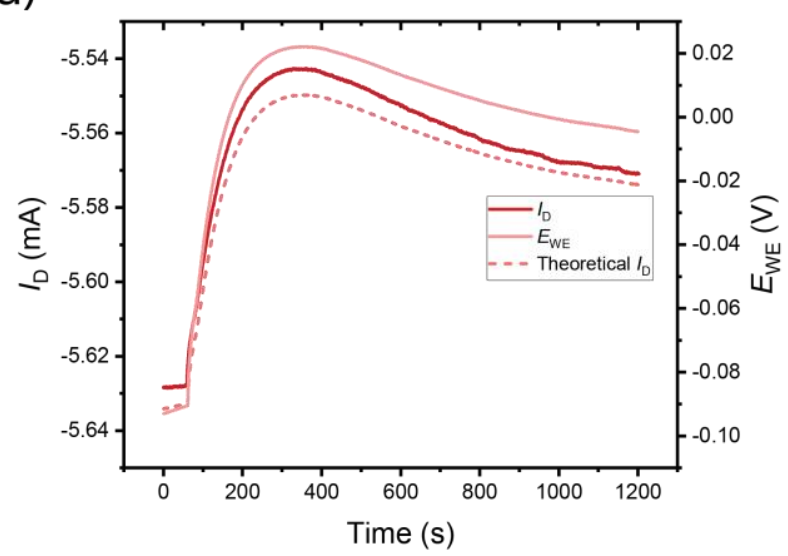

Figure S19. p(gT2) FC - PEDOT:PSS OECT L20W2. (a) $33 \mathrm{mM}$ glucose and GOx, $\mathrm{V}_{\mathrm{D}}=-0.1 \mathrm{~V}$ (b) $33 \mathrm{mM} \mathrm{H}_{2} \mathrm{O}_{2}$ and $\mathrm{PBS}, \mathrm{V}_{\mathrm{D}}=-0.1 \mathrm{~V}$ (c) $33 \mathrm{mM}$ glucose and $\mathrm{GOx}, \mathrm{V}_{\mathrm{D}}=-0.2 \mathrm{~V}$ (d) $33 \mathrm{mM} \mathrm{H}_{2} \mathrm{O}_{2}$ and PBS, $\mathrm{V}_{\mathrm{D}}=-0.2 \mathrm{~V}$ 